REPRESENTATION THEORY

An Electronic Journal of the American Mathematical Society

Volume 13, Pages 272-308 (July 7, 2009)

S 1088-4165(09)00305-7

\title{
THE BLOCKS OF THE BRAUER ALGEBRA IN CHARACTERISTIC ZERO
}

\author{
ANTON COX, MAUD DE VISSCHER, AND PAUL MARTIN
}

\begin{abstract}
We determine the blocks of the Brauer algebra in characteristic zero. We also give information on the submodule structure of standard modules for this algebra.
\end{abstract}

\section{INTRODUCTION}

The Brauer algebra $B_{n}(\delta)$ was introduced in Bra37 in the study of the representation theory of orthogonal and symplectic groups. Over $\mathbb{C}$, and for integral values of $\delta$, its action on tensor space $T=\left(\mathbb{C}^{|\delta|}\right)^{\otimes n}$ can be identified with the centraliser algebra for the corresponding group action. This generalises the Schur-Weyl duality between symmetric and general linear groups Wey46.

If $n$ is fixed, then for all $\delta \geq n$ the centraliser algebra $\operatorname{End}_{O(\delta)}(T)$ has multimatrix structure independent of $\delta$, and Brauer's algebra $B_{n}(\delta)$ unifies these algebras, having a basis independent of $\delta$, and a law of composition which makes sense over any field $k$ and for any $\delta \in k$. The Brauer algebra is well defined in particular for positive integral $\delta<n$, but the action on $T$ is faithful for positive integral $\delta$ if and only if $\delta \geq n$.

In classical invariant theory one is interested in the Brauer algebra per se only in so far as it coincides with the centraliser of the classical group action on $T$; i.e., in the case of $\delta$ integral with $|\delta|$ large compared to $n$. Here we take another view and consider the stable properties for fixed $\delta$ and arbitrarily large $n$. In such cases $B_{n}(\delta)$ is not semisimple for $\delta$ integral. However, it belongs to a remarkable family of algebras arising both in invariant theory and in statistical mechanics for which this view is very natural. (For example, when considered from the point of view of transfer matrix algebras in statistical mechanics [Mar91].) Indeed, much of the structure of $B_{n}(\delta)$ can be recovered from a suitable global limit of $n$ by localisation (and in this sense its structure does not depend on $n$ ).

This family of algebras can be introduced as follows. Consider the diagram of commuting actions on $T$, with $|\delta|=N$ :

Received by the editors December 22,2005.

2000 Mathematics Subject Classification. Primary 20G05.

(C)2009 American Mathematical Society Reverts to public domain 28 years from publication 


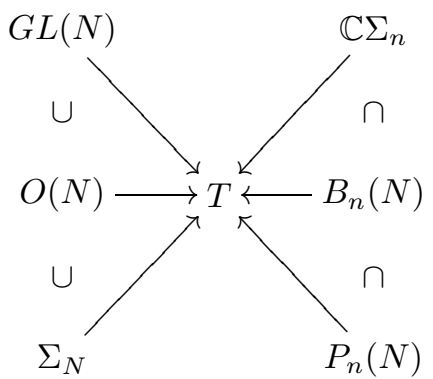

where the actions of the algebra on the right centralise the action of the group on the left in the same row, and vice versa. The bottom row consists of the diagonal action of $\Sigma_{N}$ permuting the standard ordered basis of $\mathbb{C}^{N}$ on the left, and the partition algebra $P_{n}(N)$ on the right. The partition algebra $P_{n}(\delta)$ (for any $\delta$ ) has a basis of partitions of two rows of $n$ vertices. The Brauer algebra is the subalgebra with basis the subset of pair partitions, and $\mathbb{C} \Sigma_{n}$ is the subalgebra with basis the pair partitions such that each pair contains a vertex from each row. The Brauer algebra also has a subalgebra with basis the set of pair partitions which can be represented by noncrossing lines drawn vertex-to-vertex in an interval of the plane with the rows of vertices on its boundary. This is the Temperley-Lieb algebra $T_{n}(\delta)$.

All of these algebras are rather well understood over $\mathbb{C}$, with the exception of $B_{n}$. All of their decomposition matrices are known, and all of their blocks can be described by an appropriate geometric linkage principle. For $\Sigma_{n}$ both data are trivial, since $\mathbb{C} \Sigma_{n}$ is semisimple. For $T_{n}$ each standard module has either one or two composition factors and its alcove geometry is affine $A_{1}$ (affine reflections on the real line). For $P_{n}$ each standard module has either one or two composition factors and its alcove geometry is affine $A_{\infty}$ (although locally the block structure looks like affine $A_{1}$ ).

Over $\mathbb{C}$, the Brauer algebra is semisimple for $\delta$ sufficiently large, and is generically semisimple Bro55. Hanlon and Wales studied these algebras in a series of papers HW89b, HW89a, HW90, HW94 and conjectured that $B_{n}(\delta)$ is semisimple for all non-integral choices of $\delta$. This was proved by Wenzl Wen88.

In this paper we determine the blocks of $B_{n}$ for $\delta$ integral. The simple modules of $B_{n}$ may be indexed by partitions of those natural numbers congruent to $n$ modulo 2 and not exceeding $n$, and hence by Young diagrams (if $\delta=0$, then the empty partition is omitted). We will call these indexing objects weights. Given $\delta \in R$ a ring we can associate a charge $\operatorname{ch}(\epsilon) \in R$ to each box $\epsilon$ in a Young diagram, as shown in Figure 1. We will also refer later to the usual content of boxes which, for the box $\epsilon$ in row $i$ and column $j$ is $c(\epsilon)=j-i$. It is easy to see that $c h(\epsilon)=\delta-1+2 c(\epsilon)$. For each pair of diagrams $\lambda$ and $\mu$ we will also need to consider the skew partitions $\lambda /(\lambda \cap \mu)$ and $\mu /(\lambda \cap \mu)$ consisting of those boxes occurring in $\lambda$ but not $\mu$ and in $\mu$ but not $\lambda$.

With these notations we can now state the two main results of the paper (which are valid without restriction on $\delta$ ).

Corollary 6.7. The simple modules $L(\lambda)$ and $L(\mu)$ are in the same block if and only if

(i) The boxes in $\lambda /(\lambda \cap \mu)$ (respectively $\mu /(\lambda \cap \mu)$ ) can be put into pairs whose charges sum to zero; 


\begin{tabular}{c|c|c|c}
\hline$\delta-1$ & $\delta+1$ & $\delta+3$ & $\cdots$ \\
\hline$\delta-3$ & $\delta-1$ & $\delta+1$ & $\cdots$ \\
\hline$\delta-5$ & $\delta-3$ & $\delta-1$ & $\cdots$ \\
\hline$\vdots$ & $\vdots$ & & \\
\end{tabular}

Figure 1. The charges associated to boxes in a Young diagram

(ii) if $\lambda /(\lambda \cap \mu)$ (respectively $\mu /(\lambda \cap \mu)$ ) contains $\frac{1}{-1}$ with no 1 to the right of these boxes, then it contains an even number of $1 /-1$ pairs.

Examples illustrating this result are given in Example 4.9 .

Theorem 7.3 (Summary). For any integral $\delta$ and natural number $l$ a standard module can be constructed (for some $B_{n}(\delta)$ ) whose socle series length is greater than $l$. This module also has a socle layer containing at least $l$ simple modules.

The second result shows that the structure of standard modules can become arbitrarily complicated. This is in marked contrast to the partition and TemperleyLieb algebra, and symmetric group cases.

To prove these results we use the theory of towers of recollement developed in CMPX06. This approach is already closely modelled, for $B_{n}$, in work of Doran, Wales, and Hanlon DWH99 (since both papers use the methods developed in [Mar96]). This key paper of Doran, Wales and Hanlon will be the starting point for our work, and we will generalise and refine several of their results.

The "diagram" algebras $P_{n} \supset B_{n} \supset T_{n}$ are amenable to many powerful representation theory techniques, and yet the representation theory of the Brauer algebra is highly non-trivial in comparison to the others. We shall see that, in terms of degree of difficulty, the study of Brauer representation theory in characteristic zero is at an intermediate level between the study of "classical" objects in characteristic zero and the grand theme of the representation theory of finite dimensional algebras, the study of $\Sigma_{n}$ in characteristic $p$.

Another such intermediate class of objects are the Hecke algebras of type $A$ at roots of unity, which are Ringel dual to the generalised Lie objects known as quantum groups. The Brauer algebra $B_{n}$ in characteristic zero has, through its global limit, more Lie-theory-like structure than $\Sigma_{n}$ in characteristic $p$ (for which not even a good organisational scheme within which to address the problem is known, for small primes $p$ ). This is reminiscent of the virtual algebraic Lie theory discussed for the (generalised) blob algebras in MRH04, MW03. However, in the Brauer algebra case, any candidate for an alcove geometry formulation will be considerably more complicated [Naz96, OR07]. For these reasons we consider the further study of the Brauer algebra in characteristic zero to be an important problem in representation theory.

The paper begins with a section defining the various objects of interest, and a review of their basic properties in the spirit of [CMPX06]. This is followed by a 
brief section describing some basic results about Littlewood-Richardson coefficients which will be needed in what follows. In Section 4 we begin the analysis of blocks by giving a necessary condition for two weights to be in the same block. This is based on an analysis of the action of certain central elements in the algebra on standard modules, and inductive arguments using Frobenius reciprocity. Section 5 constructs homomorphisms between standard modules in certain special cases, generalising a result in DWH99. Although not necessary for the main block result, this is of independent interest.

The classification of blocks is completed in Section 6. The main idea is to show that every block contains a unique minimal weight, and that there is a homomorphism from any standard labelled by a non-minimal weight to one labelled by a smaller weight. We also describe precisely which weights are minimal in their blocks.

In Section 7 we consider a certain explicit choice of weights, and show inductively, via Frobenius reciprocity arguments, that the corresponding standards can have arbitrarily complicated submodule structures. We conclude by outlining the modifications to our arguments required in the case $\delta=0$.

The structure of the Brauer algebra becomes much more complicated when considered over an arbitrary field $k$. For general $k$ and $\delta$ integral this algebra still acts as a centraliser algebra; this has been shown in a recent series of papers for the symplectic case [Dot98, Oeh01, DDH08, and in odd characteristic for the orthogonal case DH09. A necessary and sufficient condition for semisimplicity (which holds over arbitrary fields) was given recently by Rui [Rui05. The study of Young and permutation modules for these algebras has been started in [HP06.

Since this paper was submitted we have found a reformulation of our block result in terms of an alcove geometry of type $D[\mathrm{CDM}]$. This has inspired a new proof of the block result using symplectic Schur functors by Donkin and Tange [DT].

\section{Preliminaries}

In this section we will consider the Brauer algebra defined over a general field $k$ of characteristic $p \geq 0$, although we will later restrict attention to the case $k=\mathbb{C}$. After reviewing the definition of the Brauer algebra, we will show that families of such algebras form towers of recollement in the sense of [CMPX06] (which we will see follows from various results of Doran et al. DWH99]). This will be the framework in which we base our analysis of these algebras.

Given $n \in \mathbb{N}$ and $\delta \in k$, the Brauer algebra $B_{n}(\delta)$ is a finite dimensional associative $k$-algebra generated by certain Brauer diagrams. A general $(n, t)$-(Brauer) diagram consists of a rectangular box (or frame) with $n$ distinguished points on the northern boundary and $t$ distinguished points on the southern boundary, which we call nodes. Each node is joined to precisely one other by a line, and there may also be one or more closed loops inside the frame. Those diagrams without closed loops are called reduced. We will label the northern nodes from left to right by $1,2, \ldots, n$ and the southern nodes from left to right by $\overline{1}, \overline{2}, \ldots, \bar{t}$. We identify diagrams if they connect the same pairs of labelled nodes, and have the same number of closed loops. Lines which connect two nodes on the northern (respectively southern) boundary will be called northern (respectively southern) arcs; those connecting a northern node to a southern node will be called propagating lines. 


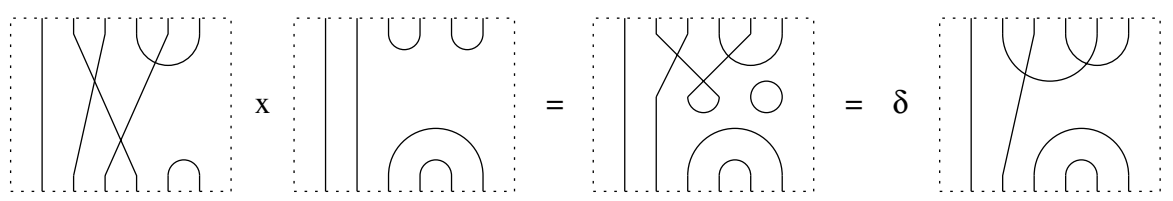

FIGURE 2. Multiplication of two diagrams in $B_{6}(\delta)$

Given an $(n, t)$-diagram $A$ and a $(t, u)$-diagram $B$, we define the product $A B$ to be the $(n, u)$-diagram obtained by concatenation of $A$ above $B$ (where we identify the southern nodes of $A$ with the northern nodes of $B$ and then ignore the section of the frame common to both diagrams). As a set, the Brauer algebra $B_{n}(\delta)$ consists of linear combinations of $(n, n)$-diagrams. This has an obvious additive structure, and multiplication is induced by concatenation. We also impose the relation that any non-reduced diagram containing $m$ closed loops equals $\delta^{m}$ times the same diagram with all closed loops removed. A basis is then given by the set of reduced diagrams. An example of a product of two diagrams is given in Figure 2. For convenience, we set $B_{0}(\delta)=k$. When no confusion is likely to arise, we denote the algebra $B_{n}(\delta)$ simply by $B_{n}$.

We will now apply as much as possible from the general setup of [CMPX06] to the Brauer algebra. The labels (A1), (A2), etc., refer to the axioms in that paper. Henceforth, we assume that $\delta \neq 0$; for the case $\delta=0$ see Section 8 .

For $n \geq 2$ consider the idempotent $e_{n}$ in $B_{n}$ defined by $1 / \delta$ times the Brauer diagram where $i$ is joined to $\bar{i}$ for $i=1, \ldots n-2$, and $n-1$ is joined to $n$ and $\overline{n-1}$ is joined to $\bar{n}$. This is illustrated in Figure 3 .

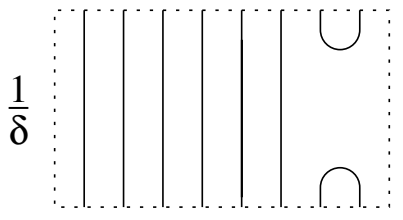

FIGURE 3. The idempotent $e_{8}$

Lemma 2.1 (A1). For each $n \geq 2$, we have an algebra isomorphism

$$
\Phi_{n}: B_{n-2} \longrightarrow e_{n} B_{n} e_{n}
$$

which takes a diagram in $B_{n-2}$ to the diagram in $B_{n}$ obtained by adding an extra northern and southern arc to the right-hand end.

This allows us to define, following Green Gre80, an exact localisation functor

$$
\begin{aligned}
F_{n}: B_{n}-\bmod & \longrightarrow B_{n-2}-\bmod \\
M & \longmapsto e_{n} M
\end{aligned}
$$

and a right exact globalisation functor

$$
\begin{aligned}
G_{n}: B_{n}-\bmod & \longrightarrow B_{n+2} \text {-mod } \\
M & \longmapsto B_{n+2} e_{n+2} \otimes_{B_{n}} M .
\end{aligned}
$$

Note that $F_{n+2} G_{n}(M) \cong M$ for all $M \in B_{n}$-mod, and hence $G_{n}$ is a full embedding. 
From this we can quickly deduce an indexing set for the isomorphism classes of simple $B_{n}$-modules. It is easy to see that

$$
B_{n} / B_{n} e_{n} B_{n} \cong k \Sigma_{n}
$$

the group algebra of the symmetric group on $n$ symbols. If the simple $k \Sigma_{n}$-modules are indexed by the set $\Lambda^{n}$, then by [Gre80] and Lemma 2.1] the simple $B_{n}$-modules are indexed by the set

$$
\Lambda_{n}=\Lambda^{n} \sqcup \Lambda_{n-2}=\Lambda^{n} \sqcup \Lambda^{n-2} \sqcup \cdots \sqcup \Lambda^{\min }
$$

where min $=0$ or 1 depending on the parity of $n$. If $p=0$ or $p>n$, then the set $\Lambda^{n}$ corresponds to the set of partitions of $n$; we write $\lambda \vdash n$ if $\lambda$ is such a partition.

For $m-n$ even we write $\Lambda_{n}^{m}$ for $\Lambda^{m}$ regarded as a subset of $\Lambda_{n}$. (If $m>n$, then $\Lambda_{n}^{m}=\emptyset$.) We also write $\Lambda$ for the disjoint union of all the $\Lambda^{n}$, and call this the set of weights for the Brauer algebra. We will henceforth abuse terminology and refer to weights as being in the same block of $B_{n}$ if the corresponding simple modules are in the same block.

For $n \geq 2$ and $0 \leq t \leq n / 2$, define the idempotent $e_{n, t}$ to be 1 if $k=0$ or $1 / \delta^{t}$ times the Brauer diagram with edges between $i$ and $\bar{i}$ for all $1 \leq i \leq n-2 t$ and between $j$ and $j+1$, and $\bar{j}$ and $\overline{j+1}$ for $n-2 t+1 \leq j \leq n-1$. (This is the image of $e_{t}$ via the isomorphism arising in Lemma 2.1.) Set $B_{n, t}=B_{n} / B_{n} e_{n, t} B_{n}$.

Lemma 2.2 (A2). The natural multiplication map

$$
B_{n, t} e_{n, t} \otimes_{e_{n, t} B_{n, t} e_{n, t}} e_{n, t} B_{n, t} \longrightarrow B_{n, t} e_{n, t} B_{n, t}
$$

is bijective. If $\delta \neq 0$ and either $p=0$ or $p>n$, then $B_{n} / B_{n} e_{n} B_{n}$ is semisimple.

Proof. The second part follows from (2.1) and standard symmetric group results. For the first part, the map is clearly surjective so we only need to show that it is also injective. It is easy to verify that:

(i) $B_{n, t}$ has a basis given by all reduced diagrams having at least $n-2 t$ propagating lines,

(ii) $B_{n, t} e_{n, t} B_{n, t}$ has a basis given by all reduced diagrams having exactly $n-2 t$ vertical edges, and

(iii) $e_{n, t} B_{n, t} e_{n, t} \cong k \Sigma_{n-2 t}$.

Now suppose that $X$ and $X^{\prime}$ are diagrams in $B_{n, t} e_{n, t}$. Any such diagram has a southern edge where the leftmost $n-2 t$ nodes lie on propagating lines, with the remaining southern nodes paired consecutively. The northern edge has exactly $t$ northern arcs. We will label such a diagram by $X_{v, 1, \sigma}$, where $v$ represents the configuration of northern arcs, 1 represents the fixed southern boundary, and $\sigma \in \Sigma_{n-2 t}$ is the permutation obtained by setting $\sigma(i)=j$ if the $i$ th propagating northern node from the left is connected to $\bar{j}$. (For later use we will denote the set of elements $v$ arising thus by $V_{n, t}$, and call such elements partial one-row diagrams.) Similarly a diagram $Y$ in $e_{n, t} B_{n, t}$ will be labelled by $Y_{1, v, \sigma}$.

It will be enough to show that the multiplication map is injective on the set of tensor products of diagram elements. Given $X=X_{v, 1, \sigma}$ and $X^{\prime}=X_{v^{\prime}, 1, \sigma^{\prime}}$ in $B_{n, t} e_{n, t}$ and $Y=Y_{1, w, \tau}, Y^{\prime}=Y_{1, w^{\prime}, \tau^{\prime}}$ in $e_{n, t} B_{n, t}$, assume that $X Y=X^{\prime} Y^{\prime}$. Then we must have $v=v^{\prime}, w=w^{\prime}$ and $\sigma \circ \tau=\sigma^{\prime} \circ \tau^{\prime}$. It now follows from the identification in (iii) that $X \otimes Y=X^{\prime} \otimes Y^{\prime}$ in $B_{n, t} e_{n, t} \otimes_{e_{n, t} B_{n, t} e_{n, t}} e_{n, t} B_{n, t}$.

We immediately obtain 
Corollary $2.3\left(\mathrm{~A} 2^{\prime}\right)$. If $\delta \neq 0$ and either $p=0$ or $p>n$, then $B_{n}$ is a quasihereditary algebra, with heredity chain given by

$$
0 \subset \cdots \subset B_{n} e_{n, k} B_{n} \subset \cdots \subset B_{n} e_{n, 0} B_{n} .
$$

The partial ordering is given as follows: for $\lambda, \mu \in \Lambda_{n}$ we have $\lambda \leq \mu$ if and only if either $\lambda=\mu$ or $\lambda \in \Lambda_{n}^{s}$ and $\mu \in \Lambda_{n}^{t}$ with $s>t$.

Henceforth we assume that $p$ satisfies the conditions in Corollary 2.3. It follows from the quasi-hereditary structure that for each $\lambda \in \Lambda_{n}$ we have a standard module $\Delta_{n}(\lambda)$ having simple head $L_{n}(\lambda)$ and all other composition factors $L_{n}(\mu)$ satisfying $\mu<\lambda$. Note that if $\lambda \in \Lambda_{n}^{n}$, then

$$
\Delta_{n}(\lambda)=L_{n}(\lambda) \cong S^{\lambda}
$$

the lift to $B_{n}$ of the Specht module for $B_{n} / B_{n} e_{n} B_{n} \cong k \Sigma_{n}$.

Note also that by [Don98, A1] and arguments as in [MRH04, Proposition 3], the quasi-hereditary structure is compatible with the globalisation and localisation functors. That is, for all $\lambda \in \Lambda_{n}$ we have

$$
\begin{aligned}
& G_{n}\left(\Delta_{n}(\lambda)\right) \cong \Delta_{n+2}(\lambda), \\
& F_{n}\left(\Delta_{n}(\lambda)\right) \cong \begin{cases}\Delta_{n-2}(\lambda) & \text { if } \lambda \in \Lambda_{n-2}, \\
0 & \text { otherwise. }\end{cases}
\end{aligned}
$$

As $F_{n}$ is exact we also have that

$$
F_{n}\left(L_{n}(\lambda)\right) \cong \begin{cases}L_{n-2}(\lambda) & \text { if } \lambda \in \Lambda_{n-2} \\ 0 & \text { otherwise }\end{cases}
$$

For every partition $\mu$ of some $m=n-2 t$ we can give an explicit construction of the modules $\Delta_{n}(\mu)$. Let $e=e_{n, t} \in B_{n}$ be as above, so that $e B_{n} e \cong B_{m}$. If we denote by $S^{\mu}$ the lift of the Specht module labelled by $\mu$ for $k \Sigma_{m}$ to $B_{m}$, then by (2.2) we have that

$$
\Delta_{n}(\mu) \cong B_{n} e \otimes_{e B_{n} e} S^{\mu} .
$$

Using this fact, it is easy to give a basis for this module in terms of some basis $\mathcal{B}(\mu)$ of $S^{\mu}$, using the notation introduced during the proof of Lemma 2.2

Lemma 2.4. If $\mu$ is a partition of $n-2 t$, then the module $\Delta_{n}(\mu)$ has a basis given by

$$
\left\{X_{v, 1, i d} \otimes x \mid v \in V_{n, t}, x \in \mathcal{B}(\mu)\right\} .
$$

Via this lemma we may identify our standard modules $\Delta_{n}(\lambda)$ with the modules $\mathcal{S}_{\lambda}(n)$ in DWH99] (which in turn come from Bro55]). Note that if we define $\Delta_{n}(\mu)$ as the tensor product in (2.5), then we have a definition that makes sense for all values of $p$. In the non-quasi-hereditary cases these modules still play an important role, as the algebras are cellular [GL96] with the $\Delta_{n}(\mu)$ as cell modules.

We will frequently need a second way to relate different Brauer algebras.

Lemma 2.5 (A3). For each $n \geq 1$, the algebra $B_{n}$ can be identified as a subalgebra of $B_{n+1}$ via the homomorphism which takes a Brauer diagram $X$ in $B_{n}$ to the Brauer diagram in $B_{n+1}$ obtained by adding two vertices $n+1$ and $\overline{n+1}$ with a line between them. 
Lemma 2.5 implies that we can consider the usual restriction and induction functors

$$
\begin{aligned}
\operatorname{res}_{n}: B_{n} \text {-mod } & \longrightarrow B_{n-1} \text {-mod, } \\
M & \left.\longmapsto M\right|_{B_{n-1}}
\end{aligned}
$$

and

$$
\begin{aligned}
\operatorname{ind}_{n}: B_{n} \text {-mod } & \longrightarrow B_{n+1} \text {-mod, } \\
M & \longmapsto B_{n+1} \otimes_{B_{n}} M .
\end{aligned}
$$

We can relate these functors to globalisation and localisation via

Lemma 2.6 (A4). (i) For all $n \geq 2$ we have that

$$
B_{n} e_{n} \cong B_{n-1}
$$

as a left $B_{n-1}$, right $B_{n-2}$-bimodule.

(ii) For all $B_{n}$-modules $M$ we have

$$
\operatorname{res}_{n+2}\left(G_{n}(M)\right) \cong \operatorname{ind}_{n}(M) .
$$

Proof. (i) Every Brauer diagram in $B_{n} e_{n}$ has an edge between $\overline{n-1}$ and $\bar{n}$. Define a map from $B_{n} e_{n}$ to $B_{n-1}$ by sending a diagram $X$ to the diagram with $2(n-1)$ vertices obtained from $X$ by removing the line connecting $\overline{n-1}$ and $\bar{n}$ and and the line from $n$, and pairing the vertex $\overline{n-1}$ to the vertex originally paired with $n$ in $X$. It is easy to check that this gives an isomorphism.

(ii) Using (i) we have

$$
\begin{aligned}
\operatorname{res}_{n+2}\left(G_{n}(M)\right) & =\left.\left(B_{n+2} e_{n+2} \otimes_{B_{n}} M\right)\right|_{B_{n+1}} \\
& \cong B_{n+1} \otimes_{B_{n}} M \cong \text { ind } M .
\end{aligned}
$$

Let $\lambda$ be a partition of $n$ and $\mu$ be a partition of $n-1$. We write $\lambda \triangleright \mu$ and $\mu \triangleleft \lambda$ if $\mu$ is obtained from $\lambda$ by removing a box from its Young diagram (equivalently if $\lambda$ is obtained from $\mu$ by adding a box to its Young diagram). Given two partitions $\lambda$ and $\mu$ of $n$, we say that $\mu$ is dominated by $\lambda$ if for all $i \geq 1$ we have

$$
\sum_{j=1}^{i} \mu_{j} \leq \sum_{j=1}^{i} \lambda_{j}
$$

Given a family of modules $M_{i}$ we will write $\biguplus_{i} M_{i}$ to denote some module with a filtration whose quotients are exactly the $M_{i}$, each with multiplicity one. This is not uniquely defined as a module, but the existence of a module with such a filtration will be sufficient for our purposes.

With the above notation we can now state the following result, which holds in arbitrary characteristic.

Proposition 2.7 (A5 and A6). (i) For $\lambda \in \Lambda_{n}$ we have short exact sequences

$$
0 \rightarrow \biguplus_{\mu \triangleleft \lambda} \Delta_{n+1}(\mu) \rightarrow \operatorname{ind}_{n} \Delta_{n}(\lambda) \rightarrow \biguplus_{\mu \triangleright \lambda} \Delta_{n+1}(\mu) \rightarrow 0
$$

and

$$
0 \rightarrow \biguplus_{\mu \triangleleft \lambda} \Delta_{n-1}(\mu) \rightarrow \operatorname{res}_{n} \Delta_{n}(\lambda) \rightarrow \biguplus_{\mu \triangleright \lambda} \Delta_{n-1}(\mu) \rightarrow 0 .
$$


(ii) In each of the filtered modules which arise in (i), the filtration can be chosen so that partitions labelling successive quotients are ordered by dominance, with the top quotient maximal among these. When $k \Sigma_{n}$ is semisimple the $\biguplus$ all become direct sums.

Proof. This was proved for $k=\mathbb{C}$ in [DWH99, Theorem 4.1 and Corollary 6.4] (as the condition $\lambda \vdash n$ in [DWH99, Corollary 6.4] is not needed), where they obtain direct sums as $\mathbb{C} \Sigma_{n}$ is semisimple. However, their proof of (i) is valid over any field, and (ii) follows from the explicit descriptions of the filtered modules in the proof of DWH99, Theorem 4.1] together with the description of induction and restriction of a Specht module for the symmetric group in [Jam78, Theorem 9.3 and 9.14].

Wenzl Wen88 has shown that $B_{n}$ is semisimple when $k=\mathbb{C}$ and $\delta \notin \mathbb{Z}$. (Over an arbitrary field, a necessary and sufficient condition for semisimplicity has been given by Rui Rui05.) For this reason we do not consider the case of non-integral $\delta$. As we will regularly need to appeal to the representation theory of the symmetric group, which is not well understood in positive characteristic, we will also only consider the characteristic zero case. In summary:

Henceforth, we will assume that $k=\mathbb{C}$ and $\delta \in \mathbb{Z} \backslash\{0\}$, unless otherwise stated.

\section{Some LitTlewood-Richardson COEFFicients}

One of the key results used by [DWH99] in their analysis of the Brauer algebra is [HW90, Theorem 4.1] which decomposes standard modules $\Delta_{n}(\lambda)$ with $\lambda \vdash n$ as symmetric group modules. Recall that a partition is even if every part of the partition is even, and that $c_{\mu \eta}^{\lambda}$ denotes a Littlewood-Richardson coefficient. If $\lambda \vdash n$ and $\mu \vdash m$, then [HW90, Theorem 4.1] states that either $\left[\operatorname{res}_{\mathbb{C} \Sigma_{n}} \Delta_{n}(\mu): S^{\lambda}\right]=0$ or $m=n-2 t$ for some $t \geq 0$ and

$$
\left[\operatorname{res}_{\mathbb{C} \Sigma_{n}} \Delta_{n}(\mu): S^{\lambda}\right]=\sum_{\substack{\eta \vdash 2 t \\ \eta \text { even }}} c_{\mu \eta}^{\lambda} .
$$

As this result is stated in terms of Littlewood-Richardson coefficients, we will find it useful to calculate these in certain special cases.

Lemma 3.1. If $\mu \subset \lambda$ are partitions such that $\nu=\lambda / \mu$ is also a partition, then

$$
c_{\mu \eta}^{\lambda}= \begin{cases}1 & \text { if } \eta=\nu, \\ 0 & \text { otherwise. }\end{cases}
$$

Proof. This follows immediately from the definition of Littlewood-Richardson coefficients in terms of rectification of skew tableaux (see [Ful97, Section 5.1, Corollary 2]).

For our second calculation we will need an alternative definition of LittlewoodRichardson coefficients (which can be found in [JK81, 2.8.14 Corollary]). When considering a configuration of boxes labelled by elements $b_{i j}$ we say that the configuration is valid if:

(i) For all $i$, if $y<j$, then $b_{i y}$ is in a later column than $b_{i j}$.

(ii) For all $j$, if $x<i$, then $b_{x j}$ is in an earlier row than $b_{i j}$. 
For each box $(i, j)$ of $\eta$ consider a symbol $b_{i j}$. Then the Littlewood-Richardson coefficient $c_{\mu \eta}^{\lambda}$ is the number of ways one can form $\lambda$ from $\mu$ by adding the boxes of $\eta$ to $\mu$ in the following manner. First add $b_{11}, b_{12}, \ldots, b_{1 \eta_{1}}$ to $\eta$ to form a new partition $\eta^{1}$. Continue inductively by adding $b_{i 1}, b_{i 2}, \ldots, b_{i \eta_{i}}$ to $\eta^{i-1}$ to form a new partition $\eta^{i}$. We require that the final configuration of the elements $b_{i j}$ is valid.

Lemma 3.2. If $\mu \subset \lambda$ are partitions with $\lambda=\left(a^{b}\right)$ for some $a$ and $b$, then there is a unique partition $\eta=\left(\eta_{1}, \ldots, \eta_{r}\right)$ such that $c_{\mu \eta}^{\lambda} \neq 0$, and for this partition we have $c_{\mu \eta}^{\lambda}=1$. Further, $(\lambda / \mu)_{i}=\eta_{r-i}$.

Proof. Consider valid extensions of $\mu$ by any $\eta$ to form $\lambda$. As $\lambda$ is a rectangle, the final row of $\eta$ can only be placed as illustrated in Figure 4. Then the penultimate row of $\eta$ must be placed as illustrated in Figure 4 . Continuing in this way we see that the choice of $\eta$ is unique, and the number of boxes in the final row of $\lambda / \mu$ must equal $\eta_{1}$, in the penultimate row must equal $\eta_{2}$, and so on.

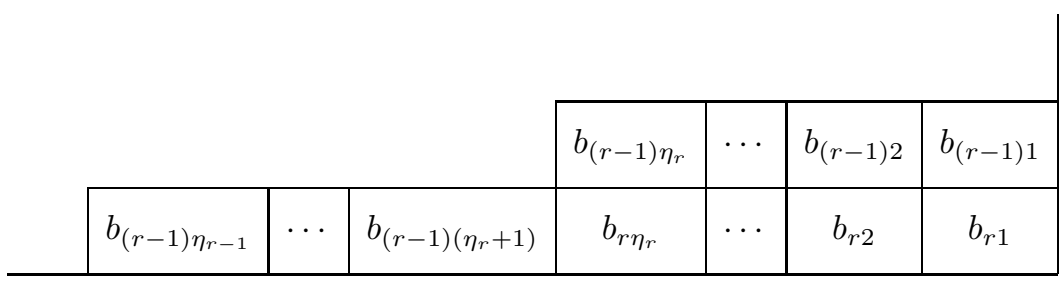

Figure 4. The final two rows of $\eta$ in $\lambda$

\section{A partial Block Result}

Doran, Wales, and Hanlon DWH99 have given a necessary condition for the existence of a non-zero homomorphism of $B_{n}$-modules from $\Delta_{n}(\lambda)$ to $\Delta_{n}(\mu)$. We will first elevate this condition to a partial block result, and then give a stronger necessary condition that must also hold for two weights to be in the same block. In Section 6 we will see that this stronger condition is also sufficient for two weights to be in the same block.

Let $\lambda$ be a partition. For a box $d$ in the corresponding Young diagram $[\lambda]$, we denote by $c(d)$ the content of $d$. Recall that if $d=(x, y)$ is in the $x$-th row (counting from top to bottom) and in the $y$-th column (counting from left to right) of $[\lambda]$, then $c(d)=y-x$. We denote by $\mathbf{c}(\lambda)$ the multiset $\{c(d): d \in[\lambda]\}$. If $\mu$ is a partition with $[\mu] \subseteq[\lambda]$ we write $\mu \subseteq \lambda$, and denote the skew partition obtained by removing $\mu$ from $\lambda$ by $\lambda / \mu$. We then denote by $\mathbf{c}(\lambda / \mu)$ the multiset $\mathbf{c}(\lambda) \backslash \mathbf{c}(\mu)$.

Write $X_{i, j}$ for the Brauer diagram in $B_{n}$ with edges between $t$ and $\bar{t}$ for all $t \neq i, j$ and with edges between $i$ and $j$ and between $\bar{i}$ and $\bar{j}$. Note that $B_{n}$ is generated by the elements $X_{i, j}$ together with the symmetric group $\Sigma_{n}$ (identified with the set of diagrams with $n$ propagating lines). We denote by $T_{n}$ the element $\sum_{1 \leq i<j \leq n} X_{i, j}$ in $B_{n}$. Recall also the definition of partial one-row diagrams in the proof of Lemma 2.2 
Lemma 4.1. Let $\mu$ be a partition of $m$ with $m=n-2 t$. For all $w \in V_{n, t}$ and $x \in S^{\mu}$ we have that

$$
T_{n}\left(X_{w, 1, i d} \otimes x\right)=\left(t(\delta-1)-\sum_{d \in[\mu]} c(d)+\sum_{1 \leq i<j \leq n}(i, j)\right)\left(X_{w, 1, i d} \otimes x\right)
$$

where $(i, j)$ denotes the element of $\Sigma_{n}$ which transposes $i$ and $j$. Hence for all $y \in \Delta_{n}(\mu)$ we have

$$
T_{n} y=\left(t(\delta-1)-\sum_{d \in[\mu]} c(d)+\sum_{1 \leq i<j \leq n}(i, j)\right) y .
$$

Proof. This is essentially [DWH99, Lemma 3.2], together with observations in the proof of [DWH99, Theorem 3.3].

The next result is a slight strengthening of [DWH99, Theorem 3.3] (which in turn generalises [Naz96, formula before (2.13)], which considers the case $\delta \in \mathbb{N}$ ). The original results provide a necessary condition for the existence of a homomorphism between two standard modules, but can be refined to prove

Proposition 4.2. Suppose that $\left[\Delta_{n}(\mu): L_{n}(\lambda)\right] \neq 0$. Then either $\lambda=\mu$ or $\lambda \in \Lambda_{n}^{r}$ and $\mu \in \Lambda_{n}^{s}$ for some $r-s=2 t>0$. Further, we must have

$$
\mu \subseteq \lambda \quad \text { and } \quad t(\delta-1)+\sum_{d \in[\lambda / \mu]} c(d)=0 .
$$

Proof. The first part of the proposition is clear from the quasi-hereditary structure of $B_{n}$. For the second part, note that by using the exactness of the localisation functor we have

$$
\left[\Delta_{n}(\mu): L_{n}(\lambda)\right]=\left[\Delta_{r}(\mu): L_{r}(\lambda)\right]
$$

and hence we may assume that $\lambda$ is a partition of $n$. In this case, $L_{n}(\lambda)=\Delta_{n}(\lambda)=$ $S^{\lambda}$, the lift of the Specht module for $\mathbb{C} \Sigma_{n}$ to $B_{n}(\delta)$, and so any Brauer diagram having fewer than $n$ propagating lines must act as zero on $L_{n}(\lambda)$. In particular, all the $X_{i, j}$ 's act as zero and hence so does $T_{n}$.

The condition that $\mu \subseteq \lambda$ now follows by regarding $\Delta_{n}(\mu)$ as a $\mathbb{C} \Sigma_{n}$-module by restriction and using (3.1), which describes the multiplicities of composition factors of such a module.

For the final condition, we know by assumption that there must exist a $B_{n^{-}}$ submodule $M$ of $\Delta_{n}(\mu)$ and a $B_{n}$-homomorphism

$$
\phi: L_{n}(\lambda) \longrightarrow \Delta_{n}(\mu) / M \text {. }
$$

Let $N$ be the $B_{n}$-submodule of $\Delta_{n}(\mu)$ containing $M$ such that

$$
\phi\left(L_{n}(\lambda)\right)=N / M
$$

As $\left.N\right|_{\mathbb{C} \Sigma_{n}}$ is semisimple, we can find a $\mathbb{C} \Sigma_{n}$-submodule $W$ of $N$ such that $N=$ $W \oplus M$ and $W \cong S^{\lambda}$. By Lemma 4.1 we have for all $y \in W$ that

$$
T_{n} y=\left(t(\delta-1)-\sum_{d \in[\mu]} c(d)+\sum_{1 \leq i<j \leq n}(i, j)\right) y .
$$

But $W \cong S^{\lambda}$ is a simple $\mathbb{C} \Sigma_{n}$-module and $\sum_{1 \leq i<j \leq n}(i, j)$ is in the centre of $\mathbb{C} \Sigma_{n}$, so it must act as a scalar on $W$. It is well known [Dia88, Chapter 1] that this 
scalar is given by $\sum_{d \in[\lambda]} c(d)$. Hence we have

$$
\begin{aligned}
T_{n} y & =\left(t(\delta-1)-\sum_{d \in[\mu]} c(d)+\sum_{d \in[\lambda]} c(d)\right) y \\
& =\left(t(\delta-1)+\sum_{d \in[\lambda / \mu]} c(d)\right) y .
\end{aligned}
$$

But $T_{n}$ must act as zero on $N$ and hence $t(\delta-1)+\sum_{d \in[\lambda / \mu]} c(d)=0$.

By standard quasi-heredity arguments [Don98, Appendix] we deduce

Corollary 4.3. Suppose that $\lambda \in \Lambda_{n}^{r}$ and $\mu \in \Lambda_{n}^{s}$ with $s<r$. If $\lambda$ and $\mu$ are in the same block, then $s=r-2 t$ for some $t \in \mathbb{N}$ and

$$
t(\delta-1)+\sum_{d \in[\lambda]} c(d)-\sum_{d \in[\mu]} c(d)=0 .
$$

When $t=2$, DWH99] gave a necessary and sufficient condition for the existence of a standard module homomorphism. From their results we obtain

Theorem 4.4. Suppose that $\mu \subset \lambda$ with $|\lambda / \mu|=2$. Then

$$
\operatorname{dim} \operatorname{Hom}\left(\Delta_{n}(\lambda), \Delta_{n}(\mu)\right) \leq 1
$$

and is non-zero if and only if $\lambda$ and $\mu$ satisfy (4.1) with $\lambda / \mu \neq\left(1^{2}\right)$. Indeed, if $\lambda / \mu=\left(1^{2}\right)$, then

$$
\left[\Delta_{n}(\mu): L_{n}(\lambda)\right]=0 .
$$

Proof. It is enough to consider the case when $\lambda \vdash n$, as the general case follows by globalisation. If $\lambda$ and $\mu$ do not satisfy the required conditions, then there is no composition factor $L_{n}(\lambda)$ in $\Delta_{n}(\mu)$ (and hence no homomorphism) by Corollary 4.3 and the remarks after [DWH99, Theorem 3.1]. In the remaining cases the existence of such a homomorphism was shown in [DWH99, Theorem 3.4]. By the remarks after [DWH99, Theorem 3.1] the multiplicity of the simple module $\Delta_{n}(\lambda)$ in $\Delta_{n}(\mu)$ is 1 , and the dimension result is now immediate.

The next result is a strengthening of Proposition 4.2

Proposition 4.5. Suppose that $\left[\Delta_{n}(\mu): L_{n}(\lambda)\right] \neq 0$. Then there is a pairing of the boxes in $\lambda / \mu$ such that the sum of the content of the boxes in each pair is equal to $1-\delta$.

Proof. We use induction on $n$; the case $n=2$ is covered by Proposition 4.2. Thus we assume that the result holds for $n-1$ and we will show that it holds for $n$.

If $\left[\Delta_{n}(\mu): L_{n}(\lambda)\right] \neq 0$, then by Proposition 4.2 we know that $\mu \subseteq \lambda$ and

$$
t(\delta-1)+\sum_{d \in[\lambda / \mu]} c(d)=0
$$

where $2 t=|\lambda|-|\mu|$. Now suppose, for a contradiction, that there is no pairing of the boxes of $[\lambda / \mu]$ satisfying the condition of the proposition. By localising we may assume that $\lambda$ is a partition of $n$, so that $L_{n}(\lambda)=\Delta_{n}(\lambda)$. Thus $\Delta_{n}(\mu)$ has a submodule $M$ such that $\Delta_{n}(\lambda) \hookrightarrow \Delta_{n}(\mu) / M$.

The partition $\lambda$ has a removable box $\epsilon_{i}$ of content $s$ say and by Proposition 2.7 we have a surjection $\operatorname{ind}_{n-1} \Delta_{n-1}\left(\lambda-\epsilon_{i}\right) \rightarrow \Delta_{n}(\lambda)$. Hence we have

$$
\operatorname{Hom}\left(\operatorname{ind}_{n-1} \Delta_{n-1}\left(\lambda-\epsilon_{i}\right), \Delta_{n}(\mu) / M\right) \neq 0
$$


and so by Frobenius reciprocity we have

$$
\operatorname{Hom}\left(\Delta_{n-1}\left(\lambda-\epsilon_{i}\right), \operatorname{res}_{n}\left(\Delta_{n}(\mu) / M\right)\right) \neq 0 .
$$

This implies that $\Delta_{n-1}\left(\lambda-\epsilon_{i}\right)=L_{n-1}\left(\lambda-\epsilon_{i}\right)$ is a composition factor of $\operatorname{res}_{n}\left(\Delta_{n}(\mu)\right)$. Now using Proposition 2.7 we see that either 0 , or

(i) the weight $\mu$ has a removable box $\epsilon_{j}$ such that $\left[\Delta_{n-1}\left(\mu-\epsilon_{j}\right): L_{n-1}\left(\lambda-\epsilon_{i}\right)\right] \neq$

(ii) the weight $\mu$ has an addable box $\epsilon_{j}$ such that $\left[\Delta_{n-1}\left(\mu+\epsilon_{j}\right): L_{n-1}\left(\lambda-\epsilon_{i}\right)\right] \neq 0$.

We consider each case in turn.

In case (i), Proposition 4.2 implies that $\left[\mu-\epsilon_{j}\right] \subseteq\left[\lambda-\epsilon_{i}\right]$ and

$$
t(\delta-1)+\sum_{d \in[\lambda / \mu]} c(d)-c\left(\epsilon_{i}\right)+c\left(\epsilon_{j}\right)=0 .
$$

Hence from (4.2) we must have

$$
c\left(\epsilon_{j}\right)=c\left(\epsilon_{i}\right)=s
$$

and by induction we can find a pairing of the boxes in $\left(\lambda-\epsilon_{i}\right) /\left(\mu-\epsilon_{j}\right)$ such that the sum of the content of the boxes in each pair is equal to $1-\delta$. But as multisets

$$
\mathbf{c}\left(\left(\lambda-\epsilon_{i}\right) /\left(\mu-\epsilon_{j}\right)\right)=\mathbf{c}(\lambda / \mu)-c\left(\epsilon_{i}\right)+c\left(\epsilon_{j}\right)=\mathbf{c}(\lambda / \mu)
$$

and hence there is such a pairing for the boxes of $\lambda / \mu$. This gives the desired contradiction.

Now consider case (ii). Here $\mu$ has an addable box $\epsilon_{j}$ such that $\left[\mu+\epsilon_{j}\right] \subseteq\left[\lambda-\epsilon_{i}\right]$ and

$$
(t-1)(\delta-1)+\sum_{d \in[\lambda / \mu]} c(d)-c\left(\epsilon_{i}\right)-c\left(\epsilon_{j}\right)=0 .
$$

Comparing with (4.2) we deduce that

$$
c\left(\epsilon_{j}\right)+c\left(\epsilon_{i}\right)=1-\delta .
$$

By induction there is a pairing of the boxes of $\left(\lambda-\epsilon_{i}\right) /\left(\mu+\epsilon_{j}\right)$ satisfying the condition of the proposition. But as multisets

$$
\mathbf{c}\left(\left(\lambda-\epsilon_{i}\right) /\left(\mu+\epsilon_{j}\right)\right)=\mathbf{c}(\lambda / \mu)-c\left(\epsilon_{i}\right)-c\left(\epsilon_{j}\right)
$$

and as observed above, the $c\left(\epsilon_{i}\right)$ and $c\left(\epsilon_{j}\right)$ can be paired in the right way. Hence the boxes of $\lambda / \mu$ can be paired appropriately, which again gives the desired contradiction.

When $\delta$ is even we will need a further refinement of Proposition 4.2. Given $\mu \subset \lambda$, consider the boxes with content $-\frac{\delta}{2}$ and $\frac{2-\delta}{2}$ in $\lambda / \mu$. If $\left[\Delta_{n}(\mu): L_{n}(\lambda)\right] \neq 0$, then these must be paired by Proposition 4.5, and so must be in one of the two chain configurations illustrated in Figure 5 (for some length of chain).

(a)

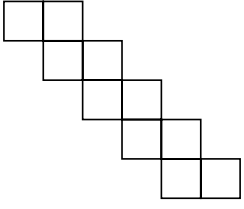

(b)

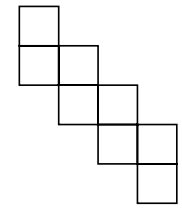

Figure 5. The two possible forms of configurations of paired boxes of contents $-\frac{\delta}{2}$ and $\frac{2-\delta}{2}$ 
Proposition 4.6. Suppose that $\left[\Delta_{n}(\mu): L_{n}(\lambda)\right] \neq 0$ and $\delta$ is even. If the boxes of content $-\frac{\delta}{2}$ and $\frac{2-\delta}{2}$ are configured in the form of Figure $5(\mathrm{~b})$, then the number of columns in this configuration must be even.

Proof. We will show by induction on $n$ that in case (b) the number of columns must be even. The case $n=2$ is covered by Theorem 4.4 .

By repeated applications of $F$ we may assume that $\lambda \vdash n$. Let $\epsilon_{i}$ be a removable box of $\lambda$. As in the proof of Proposition 4.5 we have that, if

$$
\left[\Delta_{n}(\mu): L_{n}(\lambda)\right] \neq 0,
$$

then either

$$
\left[\Delta_{n-1}\left(\mu-\epsilon_{j}\right): L_{n-1}\left(\lambda-\epsilon_{i}\right)\right] \neq 0
$$

for some removable box $\epsilon_{j}$ of $\mu$ with $c\left(\epsilon_{i}\right)=c\left(\epsilon_{j}\right)$ and $\mu-\epsilon_{j} \subset \lambda-\epsilon_{i}$, or

$$
\left[\Delta_{n-1}\left(\mu+\epsilon_{j}\right): L_{n-1}\left(\lambda-\epsilon_{i}\right)\right] \neq 0
$$

for some addable box $\epsilon_{j}$ of $\mu$ with $c\left(\epsilon_{i}\right)+c\left(\epsilon_{j}\right)=1-\delta$ and $\mu+\epsilon_{j} \subseteq \lambda-\epsilon_{i}$.

If $c\left(\epsilon_{i}\right)$ is not equal to either $-\frac{\delta}{2}$ or $\frac{2-\delta}{2}$, then the boxes of $\left(\lambda-\epsilon_{i}\right) /\left(\mu-\epsilon_{j}\right)$ (respectively of $\left.\left(\lambda-\epsilon_{i}\right) /\left(\mu+\epsilon_{j}\right)\right)$ of content $-\frac{\delta}{2}$ and $\frac{2-\delta}{2}$ are the same as those boxes in $\lambda / \mu$, and so the result follows by induction. Also, by our assumption on the configuration of such boxes the partition $\lambda$ does not have a removable box of content $\frac{2-\delta}{2}$. Thus we may assume that $\lambda$ has only one removable box $\epsilon_{i}$ of content $-\frac{\delta}{2}$ (and hence that $\lambda$ is a rectangle).

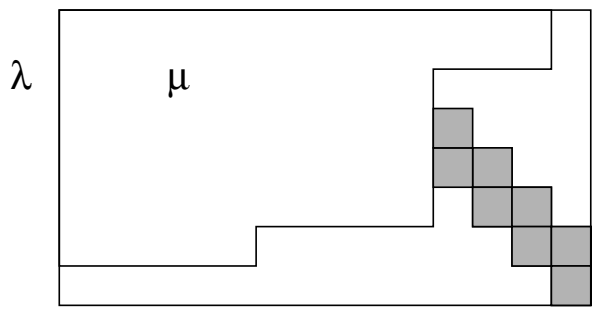

Figure 6 . The partitions $\mu \subset \lambda$, with the configuration as in Figure 5 (b) shaded

We have that $\lambda$ and $\mu$ are of the form shown in Figure $\left[\right.$ with $\left[\Delta_{n}(\mu): L_{n}(\lambda)\right] \neq$ 0 . So, in particular,

$$
\left[\operatorname{res}_{\mathbb{C} \Sigma_{n}} \Delta_{n}(\mu): S^{\lambda}\right] \neq 0
$$

By (3.1) we have

$$
\left[\operatorname{res}_{\mathbb{C} \Sigma_{n}} \Delta_{n}(\mu): S^{\lambda}\right]=\sum_{\eta \text { even }} c_{\mu \eta}^{\lambda}
$$

and hence we must have $c_{\mu \eta}^{\lambda} \neq 0$ for some even partition $\eta=\left(\eta_{1}, \ldots, \eta_{r}\right)$. As $\lambda$ is a rectangle, Lemma 3.2 implies there is only one possible $\eta$, and that each row of $\lambda / \mu$ has length $\eta_{i}$ for some $1 \leq i \leq r$. But $\eta$ was an even partition and hence these lengths are all even, which implies that the number of columns occupied by shaded boxes in Figure 6 is also even as required. 
Definition 4.7. We say that $\lambda$ and $\mu$ are $\delta$-balanced (or just balanced when the context is clear) if: (i) there exists a pairing of the boxes in $\lambda /(\lambda \cap \mu)$ (respectively in $\mu /(\lambda \cap \mu)$ ) such that the contents of each pair sum to $1-\delta$, and (ii) if $\delta$ is even and the boxes with content $-\frac{\delta}{2}$ and $\frac{2-\delta}{2}$ in $\lambda /(\lambda \cap \mu)$ (respectively in $\mu /(\lambda \cap \mu)$ ) are configured as in Figure 5(b), then the number of columns in this configuration is even.

Just as for Corollary 4.3 we can immediately deduce from Propositions 4.5] and 4.6 the following block result.

Corollary 4.8. If $\lambda$ and $\mu$ are in the same block, then they are balanced.

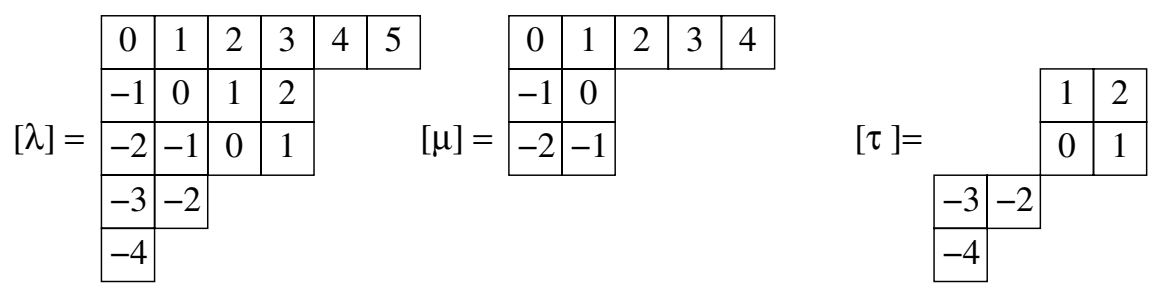

Figure 7. The diagrams $[\lambda],[\mu]$ and $[\tau]$ in Example 4.9 (i)

Example 4.9. (i) Let $\lambda=\left(6,4^{2}, 2,1\right), \mu=\left(5,2^{2}\right), \tau=\lambda /(\lambda \cap \mu)$, and $\delta=1$. The diagrams $[\lambda],[\mu]$, and $[\tau]$ are illustrated (with their contents) in Figure 7 . Clearly

$$
\sum_{d \in[\lambda]} c(d)-\sum_{d \in[\mu]} c(d)=0
$$

and hence $\lambda$ and $\mu$ satisfy the conditions in Corollary 4.3. However, there is no pairing of the boxes in $[\tau]$ such that the content of each pair sums to zero, and hence $\lambda$ and $\mu$ cannot lie in the same block.

(ii) Let $\alpha=\left(5,4^{4}\right), \beta=\left(5,1^{4}\right), \gamma=\alpha /(\alpha \cap \beta)$, and $\delta=2$. The diagrams $[\alpha]$, $[\beta]$, and $[\gamma]$ are illustrated (with their contents) in Figure 7 In this case the boxes in $[\gamma]$ can be put into pairs such that each pair sums to $1-\delta=-1$, but the boxes with contents 0 and -1 are in configuration (b) from Figure 5, and occupy an odd number of columns. Hence $\alpha$ and $\beta$ cannot lie in the same block.

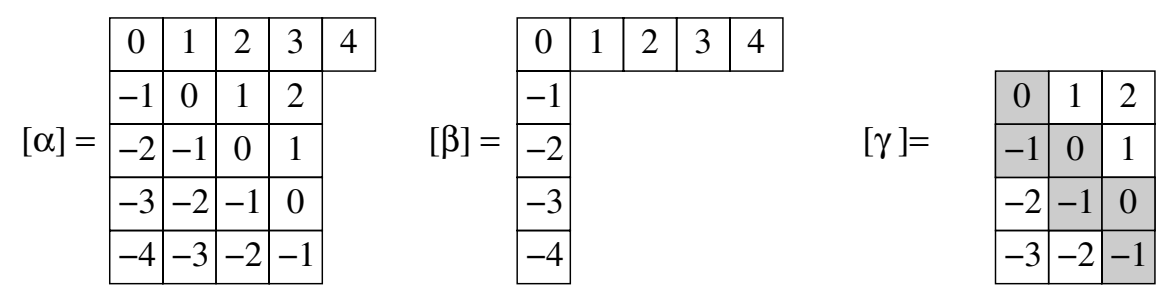

Figure 8. The diagrams $[\alpha],[\beta]$ and $[\gamma]$ in Example $4.9($ ii)

By Corollary 4.8 weights which are not balanced will lie in different blocks. Hence for a $B_{n}$-module $X$ we will denote by $\operatorname{pr}_{\lambda} X$ the direct summand of $X$ with composition factors $L_{n}(\mu)$ such that $\mu$ and $\lambda$ are balanced. 
Lemma 4.10. Suppose that $\lambda \vdash n$ and $\epsilon_{i} \in \operatorname{rem}(\lambda)$.

(i) There exists a $B_{n}$-module $X$ and a short exact sequence

$$
0 \longrightarrow X \longrightarrow \operatorname{pr}_{\lambda} \operatorname{ind}_{n-1} \Delta_{n-1}\left(\lambda-\epsilon_{i}\right) \longrightarrow \Delta_{n}(\lambda) \longrightarrow 0 .
$$

Here $X \cong \Delta_{n}\left(\lambda-\epsilon_{i}-\epsilon_{j}\right)$ if $\left(\lambda-\epsilon_{i}-\epsilon_{j}, \lambda\right)$ is a balanced pair or $X=0$ if no such $\epsilon_{j}$ exists. In the former case the sequence is non-split.

(ii) If

$$
\operatorname{Hom}\left(\operatorname{pr}_{\lambda} \operatorname{ind}_{n-1} \Delta_{n-1}\left(\lambda-\epsilon_{i}\right), \Delta_{n}(\mu)\right) \neq 0,
$$

then $\left[\Delta_{n}(\mu): L_{n}(\lambda)\right] \neq 0$.

Proof. (i) The existence of such a sequence, and the form of $X$, follows from Proposition 2.7 and Corollary 4.8. To see that the sequence is non-split, we proceed by induction on $|\lambda|$, the case where $\lambda=\emptyset$ being clear. By Frobenius reciprocity we have

$\operatorname{Hom}\left(\Delta_{n-1}\left(\lambda-\epsilon_{i}\right), \operatorname{res} \Delta_{n}\left(\lambda-\epsilon_{i}-\epsilon_{j}\right)\right) \cong \operatorname{Hom}\left(\right.$ ind $\left.\Delta_{n-1}\left(\lambda-\epsilon_{i}\right), \Delta_{n}\left(\lambda-\epsilon_{i}-\epsilon_{j}\right)\right)$

By (2.2) and Lemma 2.6)(ii) the left-hand side equals

$$
\operatorname{Hom}\left(\Delta_{n-1}\left(\lambda-\epsilon_{i}\right) \text {, ind } \Delta_{n-2}\left(\lambda-\epsilon_{i}-\epsilon_{j}\right)\right) .
$$

As $\Delta_{n-1}\left(\lambda-\epsilon_{i}\right)$ is simple, we have by the induction hypothesis and Theorem 4.4 that this Hom-space is one-dimensional. Hence the right-hand side of (4.3) is also one-dimensional, which by another application of Theorem 4.4 implies that the desired sequence is non-split as required.

(ii) Note that the head of $X$ cannot occur in the head of $\operatorname{pr}_{\lambda} \operatorname{ind}_{n-1} \Delta_{n-1}\left(\lambda-\epsilon_{i}\right)$ as $\operatorname{rad} X$ cannot be extended by $\Delta(\lambda)=L(\lambda)$. Now (ii) is an immediate consequence of (i).

\section{Computing some COMPosition multiplicities}

So far we have concentrated on conditions which imply that weights lie in different blocks of the algebra. In this section we will find certain pairs of weights which do lie in the same block, which we will demonstrate by determining certain composition factors of standard modules, and homomorphisms between such modules.

We first consider the special case where the skew partition $\lambda / \mu$ is itself a partition. For such pairs we will be able to show precisely when $L_{n}(\lambda)$ is a composition factor of $\Delta_{n}(\mu)$. We first give a necessary condition, in Proposition 5.1 which is a generalisation of [DWH99, Corollary 9.1] (the latter only considers the case $\mu=\emptyset$ and homomorphisms rather than composition factors).

Proposition 5.1. Let $\mu \subset \lambda$ be partitions such that $\nu=\lambda / \mu$ is also a partition. If

$$
\left[\Delta_{n}(\mu): L_{n}(\lambda)\right] \neq 0,
$$

then $\nu=\left(a^{b}\right)$ where $a$ is even and $b=\delta+a-1+2 c$, where $c$ is the content of the top left-hand box of $\nu$. Moreover, in this case we have

$$
\left[\Delta_{n}(\mu): L_{n}(\lambda)\right]=1 .
$$

Proof. As usual, by localisation we can assume that $\lambda$ is a partition of $n$. First suppose that $\left[\Delta_{n}(\mu): L_{n}(\lambda)\right] \neq 0$. As $L_{n}(\lambda)$ is simply the lift of $S^{\lambda}$ for $\mathbb{C} \Sigma_{n}$, we have that

$$
\left[\operatorname{res}_{\mathbb{C} \Sigma_{n}} \Delta_{n}(\mu): S^{\lambda}\right] \neq 0 .
$$


By (3.1) we have

$$
\left[\operatorname{res}_{\mathbb{C} \Sigma_{n}} \Delta_{n}(\mu): S^{\lambda}\right]=\sum_{\substack{\eta \vdash 2 k \\ \eta \text { even }}} c_{\mu \eta}^{\lambda} .
$$

Hence we see that $\nu$ must be an even partition, and by Lemma 3.1 that $\left[\Delta_{n}(\mu)\right.$ : $\left.L_{n}(\lambda)\right]=1$.

On the other hand, using Proposition 4.5 we know that there is a pairing of the boxes of $\nu$ such that the sum of the content of the boxes in each pair is equal to $1-\delta$. Clearly we have a submodule $M$ of $\Delta_{n}(\mu)$ and an embedding

$$
\Delta_{n}(\lambda) \hookrightarrow \Delta_{n}(\mu) / M .
$$

If $\epsilon_{i}$ is any removable box of $\lambda$, then we have a surjective homomorphism

$$
\operatorname{ind}_{n-1} \Delta_{n-1}\left(\lambda-\epsilon_{i}\right) \rightarrow \Delta_{n}(\lambda) .
$$

Composing these maps we see that

$$
\text { Hom }\left(\operatorname{ind}_{n-1} \Delta_{n-1}\left(\lambda-\epsilon_{i}\right), \Delta_{n}(\mu) / M\right) \neq 0
$$

and so by Frobenius reciprocity we have

$$
\operatorname{Hom}\left(\Delta_{n-1}\left(\lambda-\epsilon_{i}\right), \operatorname{res}_{n}\left(\Delta_{n}(\mu) / M\right)\right) \neq 0 .
$$

Thus

$$
\left[\operatorname{res}_{n} \Delta_{n}(\mu): L_{n-1}\left(\lambda-\epsilon_{i}\right)\right] \neq 0
$$

and hence either $\mu$ must have a removable box $\epsilon_{j}$ such that

$$
\left[\Delta_{n-1}\left(\mu-\epsilon_{j}\right): L_{n-1}\left(\lambda-\epsilon_{i}\right)\right] \neq 0
$$

or $\mu$ must have an addable box $\epsilon_{j}$ such that

$$
\left[\Delta_{n-1}\left(\mu+\epsilon_{j}\right): L_{n-1}\left(\lambda-\epsilon_{i}\right)\right] \neq 0 .
$$

In the first case we have $\mu-\epsilon_{j} \subset \lambda-\epsilon_{i}$ and hence $c\left(\epsilon_{j}\right)=c\left(\epsilon_{i}\right)$. However, as $\lambda / \mu$ is a partition this is impossible, as no removable box in $\mu$ can have the same content as some box in $\lambda / \mu$. Hence we must be in the second case with $\mu+\epsilon_{j} \subset \lambda-\epsilon_{i}$, so in fact $\epsilon_{j}$ must be a box in $\nu=\lambda / \mu$. As $\nu$ is a partition, there is only one such addable box and its content is given by $c$. Thus we must have

$$
c\left(\epsilon_{i}\right)=1-\delta-c .
$$

Now, if $\nu=\lambda / \mu$ had another removable box, then it would have to have the same content. But different removable boxes have different contents. Hence $\nu$ can only have one removable box, i.e. it is a rectangle $\nu=\left(a^{b}\right)$, where $a$ is even as $\nu$ must be an even partition. The content of the only removable box of $\nu$ inside of $\lambda$ is given by $c+a-1-(b-1)=c+a-b$ and this must be equal to $1-\delta-c$. Hence we get

$$
b=\delta-1+a+2 c
$$

as required.

We will show that the condition in Proposition 5.1 is also sufficient. This generalises DWH99, Theorem 9.2], which again only considers homomorphisms and the case $\mu=\emptyset$. Before doing this we will review some standard symmetric group results which we will require. Details can be found in [Ful97, Chapter 7]. 
We will need to consider a set of idempotents $\left\{e_{\lambda}: \lambda \vdash n\right\}$ in $\mathbb{C} \Sigma_{n}$, such that $\mathbb{C} \Sigma_{n} e_{\lambda} \cong S^{\lambda}$. We will choose

$$
e_{\lambda}=\frac{f^{\lambda}}{n !} \sum_{\sigma \in C_{\lambda}} \sum_{\tau \in R_{\lambda}} \operatorname{sgn}(\sigma) \sigma \tau
$$

where $f^{\lambda}=\operatorname{dim} S^{\lambda}, C_{\lambda}$ is the column stabiliser of $[\lambda]$ and $R_{\lambda}$ is the row stabiliser of $[\lambda]$. For example $e_{(2)}$ and $e_{(1,1)}$ (regarded as elements of $B_{2}$ ) are illustrated in Figure 9 ,

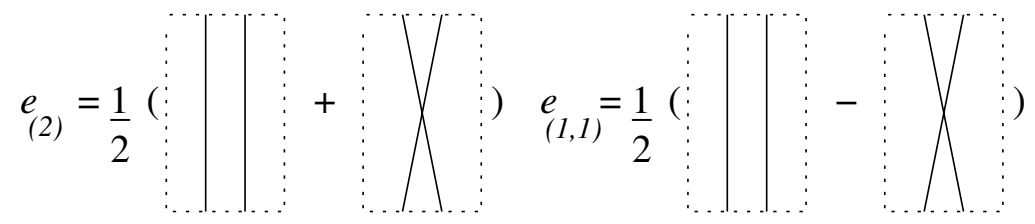

Figure 9 . The elements $e_{(2)}$ and $e_{(1,1)}$

We will also need the fact that

$$
\operatorname{ind}_{\mathbb{C}\left(\Sigma_{a} \times \Sigma_{b}\right)}^{\mathbb{C} \Sigma_{a+b}}\left(S^{\mu} \otimes S^{\nu}\right) \cong \bigoplus_{\lambda \vdash(n+m)} c_{\mu \nu}^{\lambda} S^{\lambda} .
$$

As all these group algebras are semisimple, this implies by Frobenius reciprocity that

$$
\operatorname{res}_{\mathbb{C}\left(\Sigma_{a} \times \Sigma_{b}\right)}^{\mathbb{C} \Sigma_{n}} S^{\lambda} \cong \bigoplus_{\mu \vdash a, \nu \vdash b} c_{\mu \nu}^{\lambda}\left(S^{\mu} \otimes S^{\nu}\right) .
$$

Particular values of $c_{\mu \nu}^{\lambda}$ which we will need are those where $\nu=(2)$, respectively, $\nu=(1,1)$. In these cases $c_{\mu \nu}^{\lambda}$ is at most 1 , and is non-zero precisely when $\lambda / \mu$ consists of two boxes in different columns, respectively, different rows.

Theorem 5.2. Suppose that $\mu \subset \lambda$ and $\lambda / \mu=\nu=\left(a^{b}\right)$. If $a$ is even and $b=$ $\delta-1+a+2 c$ where $c$ is the content of the top left box of $\nu$, then

$$
\left[\Delta_{n}(\mu): L_{n}(\lambda)\right]=1 .
$$

Moreover, if $\lambda \vdash n$, then

$$
\operatorname{Hom}_{B_{n}}\left(L_{n}(\lambda), \Delta_{n}(\mu)\right) \cong \mathbb{C} .
$$

Proof. We can assume, without loss of generality, that $\lambda \vdash n$. We have seen in the proof of Proposition 5.1 that $\left[\operatorname{res}_{\mathbb{C} \Sigma_{n}} \Delta_{n}(\mu): S^{\lambda}\right]=1$. Let $W=e_{\lambda} \Delta_{n}(\mu)$, which is isomorphic to $S^{\lambda}$ as a $\Sigma_{n}$-module. To show this is in fact a $B_{n}$-submodule of $\Delta_{n}(\mu)$, it will be enough to show that $X_{i, j} W=0$ for all $1 \leq i<j \leq n$. Indeed, it is enough to show that this holds for a single choice of $i$ and $j$, as

$$
\sigma X_{i, j} \sigma^{-1}=X_{\sigma(i), \sigma(j)}
$$

for all $\sigma \in \Sigma_{n}$.

So let us fix $i$ and $j$ with $1 \leq i<j \leq n$ and use the embedding

$$
\Sigma_{n-2} \times \Sigma_{2} \subset \Sigma_{n}
$$


where $\Sigma_{2}$ is the symmetric group on $\{i, j\}$ and $\Sigma_{n-2}$ the symmetric group on $\{1, \ldots, n\} \backslash\{i, j\}$. By (5.2) and the remarks following we have

$$
\operatorname{res}_{\mathbb{C}\left(\Sigma_{n-2} \times \Sigma_{2}\right)} W \cong \bigoplus_{\alpha \vdash n-2}\left(S^{\alpha} \otimes S^{(1,1)}\right) \bigoplus_{\beta \vdash n-2}\left(S^{\beta} \otimes S^{(2)}\right)
$$

where we sum over all $\alpha$ 's obtained from $\lambda$ by removing two boxes in different rows and over all $\beta$ 's obtained from $\lambda$ by removing two boxes in different columns.

The map $X_{i, j}: \Delta_{n}(\mu) \longrightarrow \Delta_{n}(\mu)$ is a $\mathbb{C} \Sigma_{n-2} \times \mathbb{C} \Sigma_{2}$-homomorphism. Note that we have $X_{i, j}\left(\Delta_{n}(\mu)\right) \subset U$ where $U$ is the span of all elements of the form $X_{w, 1, i d} \otimes x$ where $w$ has an arc between $i$ and $j$ and $x \in S^{\mu}$. Regarding $U$ as a $B_{n-2}$-module acting on the strings excluding $i$ and $j$ it is easy to see that $U$ is isomorphic to $\Delta_{n-2}(\mu)$, and the restriction of this action to $\mathbb{C} \Sigma_{n-2}$ is the same as restriction to the action of the first component of $\mathbb{C} \Sigma_{n-2} \times \mathbb{C} \Sigma_{2}$ regarded as a subalgebra of $B_{n}$. Also, it is clear that $X_{i j}$ kills the element $e_{(1,1)}$ in Figure 9, and hence kills the simple module $S^{(1,1)}$. Combining these observations with (3.1) we deduce that, as a $\mathbb{C} \Sigma_{n-2} \times \mathbb{C} \Sigma_{2}$-module, $U$ decomposes as

$$
U=\bigoplus_{\tau} c_{\tau}\left(S^{\tau} \otimes S^{(2)}\right)
$$

where

$$
c_{\tau}=\sum_{\substack{\tau \vdash n-2 \\ \eta \text { even }}} c_{\mu \eta}^{\tau}
$$

Consider the restriction $X_{i, j}: W \longrightarrow U$. We want to show that $X_{i, j} W=0$. Look at the simple summands of $W$. Every summand of the form $S^{\alpha} \otimes S^{(1,1)}$ is sent to zero as it does not appear in $U$. Moreover, if $\mu$ is not contained in $\beta$, then $S^{\beta} \otimes S^{(2)}$ is sent to zero as $U$ only contains simple modules $S^{\eta} \otimes S^{(2)}$ with $\mu \subset \eta$. So we only need to show that

$$
X_{i, j}\left(S^{\beta} \otimes S^{(2)}\right)=0
$$

for any $\beta \vdash n-2$ with $\mu \subset \beta$ and $\beta$ obtained from $\lambda$ by removing two boxes in different columns. But there is only one such $\beta$, namely the partition obtained from $\lambda$ by removing two boxes from the last row of $\nu$, i.e $\beta / \mu=\left(a^{b-1}, a-2\right)$, and by Lemma 3.1 the coefficient of $S^{\beta} \otimes S^{(2)}$ in $U$ equals 1 .

Write $W=V \oplus Y$ where $V=S^{\beta} \otimes S^{(2)}$. As $V$ is simple, either $X_{i, j}$ embeds $V$ into $U$ or $X_{i, j} V=0$. Label the boxes of the partition $\lambda$ with the numbers $1,2, \ldots, n$ starting with the first row from left to right, then the second row from left to right, etc., until the last row. Say that the last box of the partition $\nu=\left(a^{b}\right)$ inside of $\lambda$ is labelled by $l$. Up until now $X_{i, j}$ was arbitrary; we now fix $i=l-1$ and $j=l$ and we want to show that $X_{l-1, l} V=0$.

Fix a partial one-row diagram $w_{0}$ with $t$ arcs defined as follows: suppose the $u$-th row of $\nu$ inside of $\lambda$ is labelled by $x_{u}, x_{u}+1, \ldots, x_{u}+a-1$ for $1 \leq u \leq b$, as illustrated in Figure 10. Then $w_{0}$ is defined to have arcs $\left\{x_{u}, x_{u}+1\right\},\left\{x_{u}+2, x_{u}+\right.$ $3\}, \ldots\left\{x_{u}+a-2, x_{u}+a-1\right\}$ for $1 \leq u \leq b$. (Note that $x_{b}+a-1=l$.) We will represent elements of $V_{n, t}$ by adding bars to the Young tableau joining each pair of nodes connected by an arc. Thus the element $w_{0}$ will be represented by the diagram in Figure 11. Usually we will only represent the boxes of $\nu$ in such a diagram. 


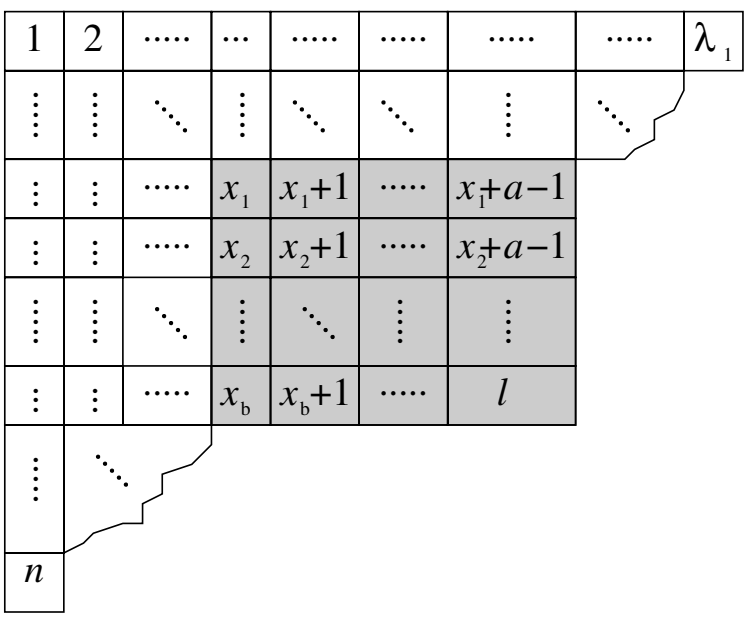

Figure 10. The labelling of $\lambda$, with $\nu$ shaded and $\mu$ unshaded

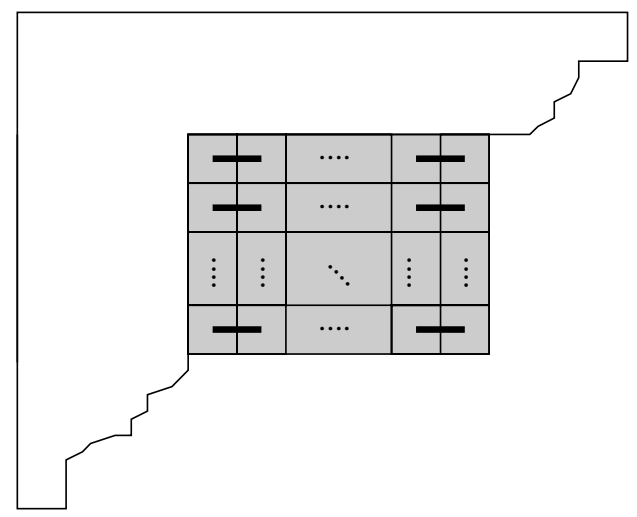

Figure 11. A diagrammatic representation of the element $w_{0}$

Now consider the element of $\Delta_{n}(\mu)$ given by $X_{w_{0}, 1, i d} \otimes x$ for some $x \in S^{\mu}$. Then $e_{\lambda}\left(X_{w_{0}, 1, i d} \otimes x\right) \in W$, so it decomposes as

$$
e_{\lambda}\left(X_{w_{0}, 1, i d} \otimes x\right)=v+y
$$

where $v \in V$ and $y \in Y$. Note that this decomposition is independent of $\delta$. As observed above, we have $X_{l-1, l} e_{\lambda}\left(X_{w_{0}, 1, i d} \otimes x\right)=X_{l-1, l} v$. Consider the coefficient of $X_{w_{0}, 1, i d} \otimes x$ in $X_{l-1, l} v$. We will show that it is a non-zero multiple of

$$
\delta-1+a-b+2 c .
$$

Hence, as $v$ is independent of $\delta$ we see that $v \neq 0$, but when $\delta-1+a-b+2 c=0$, we have $X_{l-1, l} v=0$. Thus $X_{l-1, l}$ cannot embed $V$ into $U$ and so it must map $V$ to zero.

Using the labelling of the boxes of $\lambda$ defined above, we will identify the row and column stabilisers $R_{\lambda}$ and $C_{\lambda}$ as subgroups of $\Sigma_{n}$, the symmetric group on 
$\{1, \ldots, n\}$. From (5.1) we have

$$
e_{\lambda}\left(X_{w_{0}, 1, i d} \otimes x\right)=\frac{f^{\lambda}}{n !} \sum_{\sigma \in C_{\lambda}} \sum_{\tau \in R_{\lambda}} \operatorname{sgn}(\sigma) \sigma \tau\left(X_{w_{0}, 1, i d} \otimes x\right),
$$

and so

$$
X_{l-1, l} e_{\lambda}\left(X_{w_{0}, 1, i d} \otimes x\right)=\frac{f^{\lambda}}{n !} \sum_{\sigma \in C_{\lambda}} \sum_{\tau \in R_{\lambda}} \operatorname{sgn}(\sigma) X_{l-1, l} \sigma \tau\left(X_{w_{0}, 1, i d} \otimes x\right) .
$$

We want to find the coefficient of $X_{w_{0}, 1, i d} \otimes x$ in this sum. We consider several cases.

Case 1. Suppose that $\sigma \tau X_{w_{0}, v_{k}, i d}$ has an arc $\{l-1, l\}$.

In this case $X_{l-1, l} \sigma \tau\left(X_{w_{0}, 1, i d} \otimes x\right)=\delta \sigma \tau\left(X_{w_{0}, 1, i d} \otimes x\right)$. If we want $\sigma \tau\left(X_{w_{0}, 1, i d} \otimes\right.$ $x)$ to be in $\operatorname{span}\left\{X_{w_{0}, 1, i d} \otimes S^{\mu}\right\}$, then we must have

$$
\begin{array}{ll}
\tau=\tau_{1} \tau_{2} & \text { with } \tau_{1} \in R_{\mu \subset \lambda}, \tau_{2} \in R_{\lambda}^{0}, \\
\sigma=\sigma_{1} \sigma_{2} & \text { with } \sigma_{1} \in C_{\mu \subset \lambda}, \sigma_{2} \in C_{\lambda}^{0},
\end{array}
$$

where $R_{\mu \subset \lambda}$ denotes the subgroup of $R_{\lambda}$ (isomorphic to $R_{\mu}$ ), which preserves the rows of $\mu$ and fixes everything in $\nu$ and $R_{\lambda}^{0}$ denotes the subgroup of $R_{\lambda}$, which fixes $X_{w_{0}, 1, i d}$ as a diagram (i.e. fixes all but the $t$ northern arcs, which may be permuted amongst themselves and be reversed). In a similar way we define $C_{\mu \subset \lambda}$ and $C_{\lambda}^{0}$.

Set $r=\left|R_{\lambda}^{0}\right|$. As the $a$ columns of $\nu$ are paired by the bars in $w_{0}$, and each pair of such columns may be permuted freely by $C_{\lambda}^{0}$ we have $\left|C_{\lambda}^{0}\right|=(b !)^{a / 2}$. Moreover, $\operatorname{sgn}\left(\sigma_{2}\right)=1$ as $\sigma_{2}$ is an even permutation (as it is made up of pairs of identical permutations, corresponding to the paired ends of a bar) and $\operatorname{so} \operatorname{sgn}(\sigma)=\operatorname{sgn}\left(\sigma_{1}\right)$. Hence in this case we get the contribution

$$
\begin{aligned}
& \frac{f^{\lambda}}{n !} \sum_{\sigma_{2} \in C_{\lambda}^{0}} \sum_{\sigma_{1} \in C_{\mu \subset \lambda}} \sum_{\tau_{2} \in R_{\lambda}^{0}} \sum_{\tau_{1} \in R_{\mu \subset \lambda}} \operatorname{sgn}\left(\sigma_{1} \sigma_{2}\right) \sigma_{1} \sigma_{2} \tau_{1} \tau_{2}\left(X_{w_{0}, 1, i d} \otimes x\right) \\
& =\frac{f^{\lambda}}{n !} \sum_{\sigma_{2} \in C_{\lambda}^{0}} \sum_{\tau_{2} \in R_{\lambda}^{0}} \sigma_{2} \tau_{2}\left(X_{w_{0}, 1, i d} \otimes \sum_{\sigma_{1} \in C_{\mu \subset \lambda}} \sum_{\tau_{1} \in R_{\mu \subset \lambda}} \operatorname{sgn}\left(\sigma_{1}\right) \sigma_{1} \tau_{1}(x)\right) \\
& =\frac{f^{\lambda}}{n !} \frac{|\mu|}{f^{\mu}} \sum_{\sigma_{2} \in C_{\lambda}^{0}} \sum_{\tau_{2} \in R_{\lambda}^{0}} \sigma_{2} \tau_{2}\left(X_{w_{0}, 1, i d} \otimes e_{\mu}(x)\right) \\
& =\frac{f^{\lambda}}{n !} \frac{|\mu|}{f^{\mu}} r(b !)^{a / 2}\left(X_{w_{0}, 1, i d} \otimes x\right)
\end{aligned}
$$

using for the second equality for the isomorphisms $C_{\mu \subset \lambda} \cong C_{\mu}$ and $R_{\mu \subset \lambda} \cong R_{\mu}$, and for the final equality the fact that $e_{\mu}(x)=x$ for all $x \in S^{\mu}$.

Case 2. Suppose that neither $l-1$ nor $l$ is part of an arc in $\sigma \tau X_{w_{0}, 1, i d}$.

In this case $X_{l-1, l} \sigma \tau X_{w_{0}, 1, i d}$ has $t+1$ arcs in the top row and so $X_{l-1, l}\left(X_{w_{0}, 1, i d} \otimes\right.$ $x)=0$.

Case 3. Suppose that in $\sigma \tau X_{w_{0}, v_{k}, i d}$ there are arcs $\{l-1, i\}$ and $\{l, j\}$.

In this case, $X_{l-1, l} \sigma \tau X_{w_{0}, 1, i d}$ is obtained from $\sigma \tau X_{w_{0}, 1, i d}$ by replacing the arcs $\{l-1, i\}$ and $\{l, j\}$ by the $\operatorname{arcs}\{i, j\}$ and $\{l-1, l\}$. Hence if we want to have $X_{l-1, l} \sigma \tau\left(X_{w_{0}, 1, i d} \otimes x\right)$ lying in span $\left\{X_{w_{0}, 1, i d} \otimes S^{\mu}\right\}$, then $\{i, j\}$ must be an arc of $w$ and $i=j \pm 1$. Here we consider two subcases. 
Subcase (3a). First assume that the pair $\{i, j\}$ is not in the last double column. Then $\tau=\tau_{2} \tau_{1}$ with $\tau_{1} \in R_{\mu \subset \lambda}$ and $\tau_{2} \in \tilde{\tau} R_{\lambda}^{0}$, where $\tilde{\tau}=(u-1, v)$ or $(u, v)$ such that $v$ is a box of $\nu$ in the same column as $l$ (possibly $l$ itself) and $u$ is the box of $\nu$ in the same row as $v$ and in the same column as $\max (i, j)$. An example of such a situation is illustrated in Figure 12

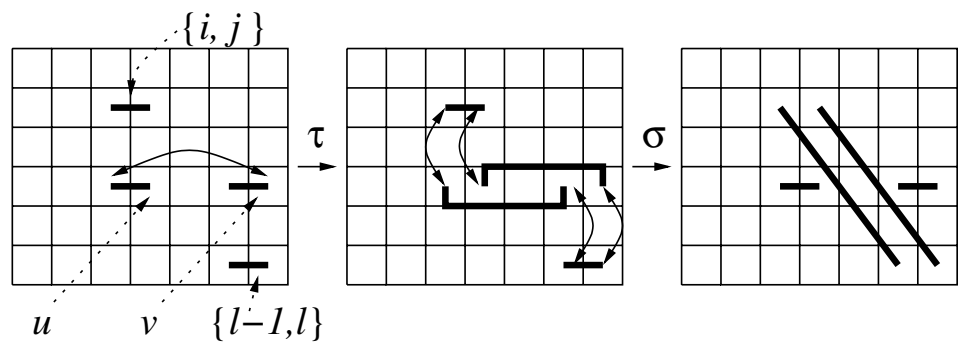

Figure 12. An example of Subcase 3(a)

Thus we have $b$ choices for $v$ and $\left(\frac{a}{2}-1\right)$ choices for the position of $\{i, j\}$ (and hence of $u)$, and so there are $2 b\left(\frac{a}{2}-1\right)$ choices for $\tilde{\tau}$. Hence there are $2 r b\left(\frac{a}{2}-1\right)$ choices for $\tau_{2}$. Now $\sigma=\sigma_{2} \sigma_{1}$ where $\sigma_{1} \in C_{\mu \subset \lambda}$, and $\sigma_{2}$ permutes the pairs in all double columns, except the last and the double column containing $\{j-1, j\}$, arbitrarily. In the last double column it must send $v-1$ to $l-1$ and $v$ to $l$, and in the double column containing $\{j-1, j\}$, it can permute the pairs in any way (as $\{j-1, j\}$ can be any pair in this double column). So we get $(b !)^{\frac{a}{2}-2}(b-1) ! b !$ possibilities for $\sigma_{2}$. Note also that $\sigma_{2}$ is always an even permutation and $\operatorname{so} \operatorname{sgn}(\sigma)=\operatorname{sgn}\left(\sigma_{1}\right)$. Thus in this subcase, we get a contribution of

$$
\begin{aligned}
& \frac{f^{\lambda}}{n !} 2 r b\left(\frac{a}{2}-1\right)(b !)^{\left(\frac{a}{2}-2\right)}(b-1) ! b ! X_{w_{0}, 1, i d} \otimes \sum_{\sigma_{1} \in C_{\mu \subset \lambda}} \sum_{\tau_{1} \in R_{\mu \subset \lambda}} \operatorname{sgn}\left(\sigma_{1}\right) \sigma_{1} \tau_{1}(x) \\
& =\frac{f^{\lambda}}{n !} \frac{|\mu| !}{f^{\mu}} r(a-2)(b !)^{\frac{a}{2}} X_{w_{0}, 1, i d} \otimes x
\end{aligned}
$$

where the equality follows as in Subcase 1.

Subcase 3(b). Next assume that the pair $\{i, j\}$ is in the last column. We must have $\tau=\tau_{2} \tau_{1}$ where $\tau_{1} \in R_{\mu \subset \lambda}$ and $\tau_{2} \in R_{\lambda}^{0}$. Also $\sigma=\sigma_{2} \sigma_{1}$ where $\sigma_{1} \in C_{\mu \subset \lambda}$ and $\sigma_{2} \in(j, l) C_{\lambda}^{0}$. We have $b-1$ choices for $j$ being a box of $\nu$ in the same column as $l$. Note that in this case $\operatorname{sgn}\left(\sigma_{2}\right)=-1$ and $\operatorname{so} \operatorname{sgn}(\sigma)=-\operatorname{sgn}\left(\sigma_{1}\right)$. Hence arguing as in Subcases 1 and 3(a) we get a contribution of

$$
-\frac{f^{\lambda}}{n !} \frac{|\mu| !}{f^{\mu}} r(b-1)(b !)^{\frac{a}{2}} X_{w_{0}, 1, i d} \otimes x .
$$

Case 4. Suppose that in $\sigma \tau X_{w_{0}, 1, i d}$ there is a link from $l-1$ to $i$, say, and $l$ is not part of an arc (or vice versa).

In this case $X_{l-1, l} \sigma \tau X_{w_{0}, 1, i d}$ is obtained from $\sigma \tau X_{w_{0}, 1, i d}$ by replacing the arc $\{i, l-1\}$ (or $\{i, l\}$ ) with the arc $\{l-1, l\}$ and $i$ is no longer part of an arc. So, if we want to have $X_{l-1, l} \sigma \tau X_{w_{0}, 1, i d}$ in $\operatorname{span}\left\{X_{w_{0}, 1, i d} \otimes S^{\mu}\right\}$, then $i$ cannot be one of the boxes of $\nu$. There are various potential subcases that can arise. After action by an element of $R_{\mu \subset \lambda}$ the element $i$ may be in any box in the same row of $\mu$. There are 
three cases: (a) $i$ is now to the left of the first column of $\nu$; (b) $i$ is above $\nu$ but not above $l-1$ or $l$; (c) $i$ is above $l-1$ or $l$.

Subcase 4(a). First, assume that the box $i$ is in a column to the left of $\nu$ in $\lambda$. In this case, $\tau=\tau_{2} \tau_{1}$ where $\tau_{1} \in R_{\mu \subset \lambda}$ (as we have already acted by such an element to put $i$ in this case above) and $\tau_{2} \in(v-1, u) R_{\lambda}^{0}$, or $\tau_{2} \in(v, u) R_{\lambda}^{0}$ where $v$ is any box in $\nu$ in the same column as $l$ and $u$ is the box of $\mu$ in the same row as $v$ and in the same column as $i$. An example of such a situation is illustrated in Figure 13 .

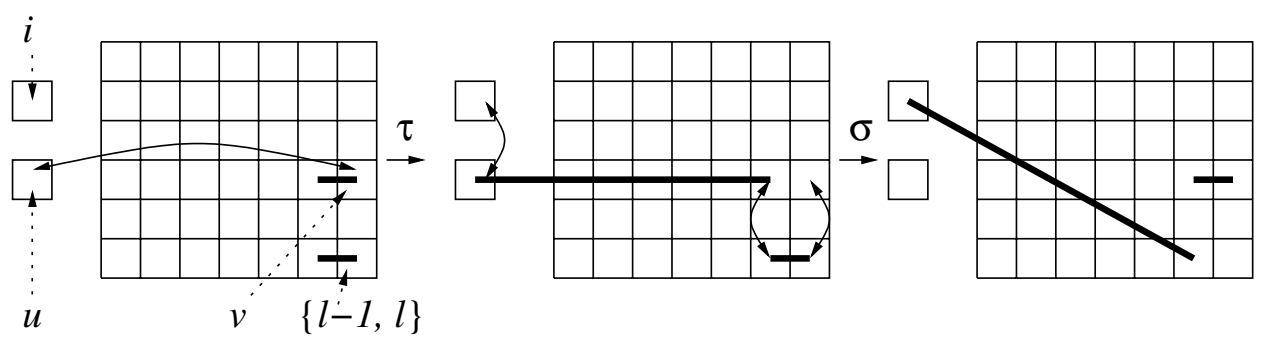

Figure 13. An example of Subcase 4(a)

Let $c_{1}$ be the number of columns of $\lambda$ to the left of $\nu$. Then there are $2 r b c_{1}$ possible choices of $\tau_{2}$. Now $\sigma=\sigma_{2} \sigma_{1}$ where $\sigma_{1} \in C_{\mu \subset \lambda}$ (as $i$ is an arbitrary element in its column of $\mu$ ) and $\sigma_{2}$ permutes the pairs in each of the first $\left(\frac{a}{2}-1\right)$ double columns of $\nu$ arbitrarily, and in the last double column sends $v-1$ to $l-1$ and $v$ to $l$ and then permutes the other pairs arbitrarily. Note that $\operatorname{sgn}\left(\sigma_{1}\right)=\operatorname{sgn}(\sigma)$. Hence (arguing as in earlier cases) we get a contribution of

$$
\begin{aligned}
& \frac{f^{\lambda}}{n !} \frac{|\mu| !}{f^{\mu}} 2 r b c_{1}(b !)^{\left(\frac{a}{2}-1\right)}(b-1) ! X_{w_{0}, 1, i d} \otimes x \\
& =\frac{f^{\lambda}}{n !} \frac{|\mu| !}{f^{\mu}} r(b !)^{\frac{a}{2}} 2 c_{1} X_{w_{0}, 1, i d} \otimes x .
\end{aligned}
$$

Subcase 4(b). Suppose that $i$ is a box of $\mu$ which is above some column of $\nu$ but to the left of $l-1$. Then the only way to use row and column permutations not involving $R_{\mu \subset \lambda}$ (which we have already used to position $i$ ) to connect $i$ and $l$ (or $l-1)$ is by some pair $\tau$ and $\sigma$ similar to that shown in Figure 14. But (as illustrated) any such pair does not preserve the remaining edges in $\nu$. Hence this subcase cannot arise.

Subcase $4(\mathrm{c})$. Finally, we are left with the subcase where after action by $R_{\mu \subset \lambda}$ the element $i$ is in a box of $\mu$ which is either in the same column as $l-1$ or in the same column as $l$. In this case $\tau=\tau_{2} \tau_{1}$ where $\tau_{1} \in R_{\mu \subset \lambda}$ and $\tau_{2} \in R_{\lambda}^{0}$. Also, $\sigma=\sigma_{2} \sigma_{1}$ where $\sigma_{1} \in C_{\mu \subset \lambda}$ (as $i$ is an arbitrary element in its column of $\mu$ ) and either $\sigma_{2} \in(i, l) C_{\lambda}^{0}$ or $\sigma_{2} \in(i, l-1) C_{\lambda}^{0}$. If $c_{2}$ is the number of columns above $\nu$ in $\lambda$, then there are $2 c_{2}$ choices for the position of $i$. Note that here $\operatorname{sgn}\left(\sigma_{2}\right)=-1$ and so $\operatorname{sgn}(\sigma)=-\operatorname{sgn}\left(\sigma_{1}\right)$. Hence, in this case we get a contribution of

$$
-\frac{f^{\lambda}}{n !} \frac{|\mu| !}{f^{\mu}} r(b !)^{\frac{a}{2}} 2 c_{2} X_{w_{0}, 1, i d} \otimes x .
$$



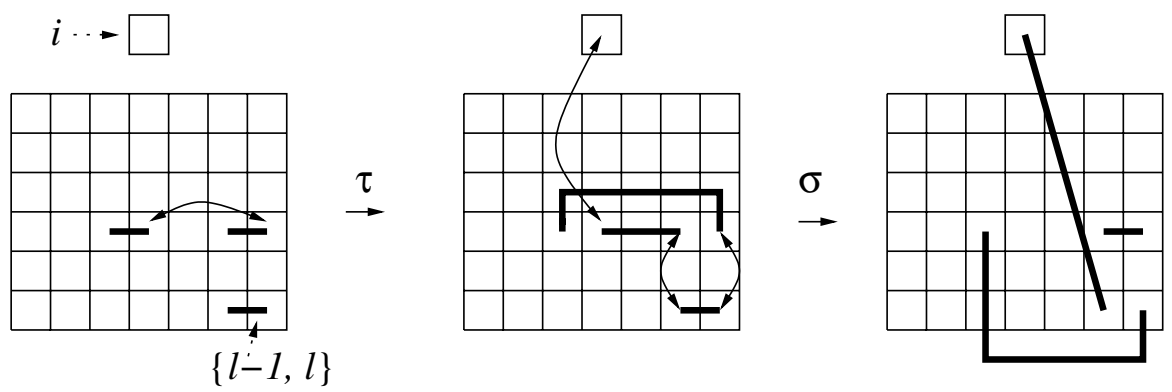

FIgURE 14. An example of the impossibility of subcase 4(b)

Note that the final sets of permutations obtained in Subcases 4(a) and 4(c) are disjoint, so there is no double counting in these contributions. Now by adding up all contributions from Cases 1-4 we see that the coefficient of $X_{w_{0}, 1, i d} \otimes x$ inside of $X_{l-1, l} e_{\lambda}\left(X_{w_{0}, 1, i d} \otimes x\right)$ is given by

$$
\frac{f^{\lambda}}{n !} \frac{|\mu| !}{f^{\mu}} r(b !)^{\frac{a}{2}}\left(\delta-1+a-b+2\left(c_{1}-c_{2}\right)\right) .
$$

The content of the top left box of the partition $\nu$ inside the partition $\lambda$ is given by $c=\left(c_{1}+1\right)-\left(c_{2}+1\right)=c_{1}-c_{2}$. Thus we have proved that this coefficient is a non-zero multiple of $(\delta-1)+a-b+2 c$ as required.

\section{The Blocks of the Brauer algebra}

In Section 4 we saw that a necessary condition for two weights $\lambda$ and $\mu$ to be in the same block was that the pair was balanced. We will now show that this condition is also sufficient. The key idea will be to construct from any partition $\lambda$ in a balanced pair with some $\mu \subset \lambda$ a partition $\nu \subset \lambda$ and a homomorphism connecting $\Delta_{n}(\lambda)$ and $\Delta_{n}(\nu)$. This will allow us to proceed by induction.

Given a partition $\lambda$ we denote by $\operatorname{add}(\lambda)$ the set of addable boxes of $\lambda$ (i.e. the set of boxes which may be added to $\lambda$ such that the new shape is still a partition). Similarly, we denote by $\operatorname{rem}(\lambda)$ the set of removable boxes of $\lambda$. If $\mu \subset \lambda$, then we denote the set of boxes in $\operatorname{rem}(\lambda)$ which are also boxes of $\lambda / \mu$ by $\operatorname{rem}(\lambda / \mu)$. Distinct boxes in $\operatorname{add}(\lambda)$ (respectively in $\operatorname{rem}(\lambda)$ ) have distinct contents, and we will identify such boxes by their contents. We will order the boxes in $\lambda$ with a given content by saying that box $\epsilon$ is smaller than box $\epsilon^{\prime}$ if $\epsilon$ appears on an earlier row than $\epsilon^{\prime}$.

Definition 6.1. Suppose that $\mu \subset \lambda$ is a balanced pair. For each $\epsilon_{i} \in \operatorname{rem}(\lambda / \mu)$ we wish to consider $\mu^{i}$, the $i$-maximal balanced subpartition between $\mu$ and $\lambda$. This is the maximal partition $\mu^{i} \subset \lambda$ such that $\mu^{i}$ does not contain $\epsilon_{i}$ and $\lambda$ and $\mu^{i}$ form a balanced pair. We will construct $\mu^{i}$ by recursively defining a series of skew partitions $\left(\lambda / \mu^{i}\right)_{j}$ which will eventually equal the skew partition $\lambda / \mu^{i}$. There is by the pairing condition a maximal box (i.e. all others smaller) with content $c\left(\epsilon_{i}^{\prime}\right)$ such that $c\left(\epsilon_{i}\right)+c\left(\epsilon_{i}^{\prime}\right)=1-\delta$. Let $\left(\lambda / \mu^{i}\right)_{0}=\left\{\epsilon_{i}, \epsilon_{i}^{\prime}\right\}$. Given $\left(\lambda / \mu^{i}\right)_{m}$, we set

$$
\left(\lambda / \mu^{i}\right)_{m+1}=\left(\lambda / \mu^{i}\right)_{m} \cup A_{m+1} \cup A_{m+1}^{\prime}
$$

where $A_{m+1}$ is the set of boxes $\epsilon$ in $\lambda$ such that $\epsilon$ is to the right of or below a box in $\left(\lambda / \mu^{i}\right)_{m}$, and $A_{m+1}^{\prime}$ is the set of boxes $\epsilon^{\prime}$ in $(\lambda / \mu)$ such that $c(\epsilon)+c\left(\epsilon^{\prime}\right)=1-\delta$ 
for some $\epsilon \in A_{m+1}$ and $\epsilon^{\prime}$ is maximal with such content among the boxes of $\lambda / \mu$ not already in $\left(\lambda / \mu^{i}\right)_{m}$.

This iterative process eventually stabilises, and we obtain $\left(\lambda / \mu^{i}\right)_{t}$ which is a (possibly disconnected) subset of the edge of $\lambda / \mu$, having width one. (In particular, it does not contain two boxes with the same content.) If $\delta$ is even and $\left(\lambda / \mu^{i}\right)_{t}$ does not contain a vertical pair of boxes with content $\frac{2-\delta}{2}$ and $-\frac{\delta}{2}$, or $\delta$ is odd and $\left(\lambda / \mu^{i}\right)_{t}$ does not contain a box of content $\frac{1-\delta}{2}$, then we set $\lambda / \mu^{i}=\left(\lambda / \mu^{i}\right)_{t}$. Otherwise if $\delta$ is even we set

$$
\left(\lambda / \mu^{i}\right)_{t+1}=\left(\lambda / \mu^{i}\right)_{t} \cup\{x, y\}
$$

where $x, y$ are the maximal boxes in $\lambda$ of content $\frac{2-\delta}{2}$ and $-\frac{\delta}{2}$ not in $\left(\lambda / \mu^{i}\right)_{t}$, and if $\delta$ is odd we set

$$
\left(\lambda / \mu^{i}\right)_{t+1}=\left(\lambda / \mu^{i}\right)_{t} \cup\{z\}
$$

where $z$ is the maximal box in $\lambda$ of content $\frac{1-\delta}{2}$ not in $\left(\lambda / \mu^{i}\right)_{t}$. This new skew partition is not necessarily stable under the addition of boxes $A$ and $A^{\prime}$ as above, and we repeat that process again until the skew partition eventually stabilises at some step $s$. We then set $\lambda / \mu^{i}=\left(\lambda / \mu^{i}\right)_{s}$. Thus $\lambda / \mu^{i}$ is a removable subset of $\lambda / \mu$ having width at most two (so at most two boxes with any given content).

(a)

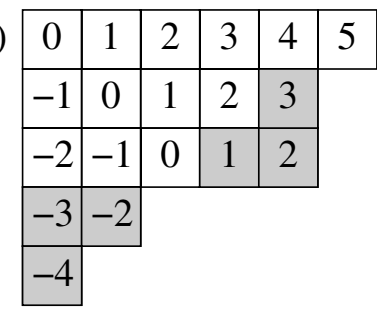

\begin{tabular}{|c|c|c|c|c|c|c|}
\hline 0 & 1 & 2 & 3 & 4 & 5 & 6 \\
\hline-1 & 0 & 1 & 2 & 3 & 4 & \\
\hline-2 & -1 & 0 & 1 & 2 & & \\
\hline-3 & -2 & -1 & 0 & 1 & & \\
\hline-4 & -3 & & & & & \\
\hline-5 & -4 & & & & & \\
\hline
\end{tabular}

(b)

Figure 15. Two examples of the $\lambda / \mu^{i}$ construction

Example 6.2. We will now consider several examples of this construction. First let $\lambda=(6,5,5,2,1)$ and $\mu=(6,4,1)$; this is a balanced pair for $\delta=2$. If $\epsilon_{i}$ is any of the boxes in $\operatorname{rem}(\lambda / \mu)$ in Figure 15(a), then $\lambda / \mu^{i}$ is the shaded region shown. For an example where the resulting skew partition is connected, consider $\lambda=(7,6,5,5,2,2)$ and $\mu=(7,4,4,1,1)$. This is a balanced pair for $\delta=2$. If $\epsilon_{i}$ is any of the removable boxes in $\lambda / \mu$, then the skew partition $\lambda / \mu^{i}$ is the shaded region shown in Figure 15(b). In this case there is a pair of boxes in the skew partition with contents $\frac{2-\delta}{2}$ and $-\frac{\delta}{2}$ (i.e. 0 and -1 ), but we do not get a strip of width 2 because these boxes are not vertically aligned.

For an example of the full iterative process consider $\lambda=\left(7,6,4^{4}, 1^{2}\right)$ and $\mu=$ $\left(5,2^{4}\right)$. This is a balanced pair for $\delta=2$, and after the first part of the iterative process the skew partition stabilises into the lightly shaded region shown in Figure [16(a). However, we now have a vertical pair in the skew partition with contents $\frac{2-\delta}{2}$ and $-\frac{\delta}{2}$ (i.e. 0 and -1 ). Thus we have to apply (6.1), and add the darkly shaded boxes with content 0 and -1 to this skew partition. The complement of this is no longer a partition, so we remove the remaining darkly shaded region by one further application of the iterative procedure. 
(a)

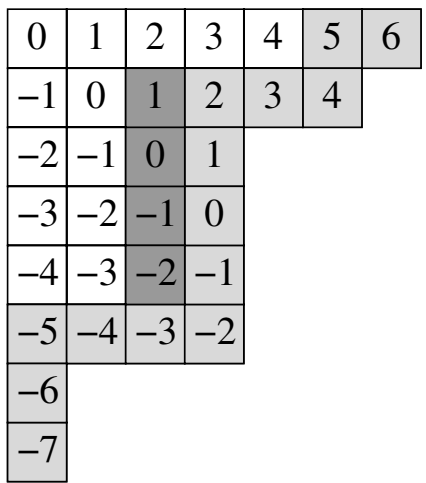

(b)

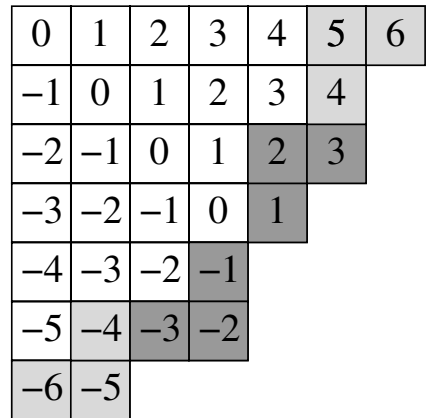

FiguRE 16. More examples of the $\lambda / \mu^{i}$ construction

Definition 6.3. We now wish to define a maximal balanced subpartition between $\mu$ and $\lambda$, which we will denote by $\mu^{\prime}$. Having constructed a skew partition $\lambda / \mu^{i}$ for each removable box $\epsilon_{i}$ of $\lambda$, we partially order this collection by inclusion. We then take $\lambda / \mu^{\prime}$ to be some minimal element of this set.

Example 6.4. For a non-trivial example of this choice, consider $\lambda=\left(7,6^{2}, 5,4^{2}, 2\right)$ and $\mu=\left(5,3,2^{3}, 1\right)$. This is a balanced pair for $\delta=1$, but has several different associated skew partitions. If we take $\epsilon_{i}$ to be one of the removable boxes labelled by 6 or -5 , then $\lambda / \mu^{i}$ equals the entire shaded region in Figure 16(b). However, if we take $\epsilon_{j}$ to be any of the other removable boxes, then $\lambda / \mu^{j}$ consists of the six darkly shaded boxes. As $\lambda / \mu^{j} \subset \lambda / \mu^{i}$, we take $\lambda / \mu^{\prime}$ to equal $\lambda / \mu^{j}$ in this case, and hence $\mu^{\prime}=\left(7,6,4^{2}, 3,2^{2}\right)$. (Note that if this example had one additional box of content 0 between the two darkly shaded regions, then we would have to apply (6.2) and this box would have associated skew partition all of the darkly shaded region together with itself and the diagonally adjacent box with content 0 .)

The importance of this construction is given by

Theorem 6.5. If $\mu \subset \lambda$ is a balanced pair, then for any maximal balanced subpartition $\mu^{\prime}$ between $\mu$ and $\lambda$ we have

$$
\operatorname{Hom}\left(\Delta_{n}(\lambda), \Delta_{n}\left(\mu^{\prime}\right)\right) \neq 0 .
$$

Proof. As usual, we may assume that $\lambda$ is a partition of $n$. Pick $\epsilon \in \operatorname{rem}\left(\lambda / \mu^{\prime}\right)$ with $\left|c(\epsilon)-\frac{1-\delta}{2}\right|$ maximal. (Note that there are at most two such boxes.) If $\delta$ is even and $c(\epsilon)=\frac{-\delta}{2}$ or $c(\epsilon)=\frac{2-\delta}{2}$, then $\lambda / \mu^{\prime}$ is one of the two cases in Figure 17(a) or (b), while if $\delta$ is odd and $c(\epsilon)=\frac{1-\delta}{2}$, then $\lambda / \mu^{\prime}$ is as in Figure 17(c). In each of these cases there is a non-zero homomorphism from $\Delta_{n}(\lambda)$ to $\Delta_{n}\left(\mu^{\prime}\right)$ by Theorem 5.2 (or more directly by repeated applications of Frobenius reciprocity). Thus we henceforth assume we are not in any of these cases.

Suppose that $\epsilon$ is paired with a maximal $\epsilon^{\prime}$ of content $1-\delta-c(\epsilon)$. We will assume that $\epsilon$ is above, or to the right of, $\epsilon^{\prime}$, and leave the (obvious) modifications required for the other case to the reader.

Note that if $\left|\lambda / \mu^{\prime}\right|=2$, then we are done by Theorem 4.4 We will be able to proceed by induction on $\left|\lambda / \mu^{\prime}\right|$ using the following claim. 
(a)

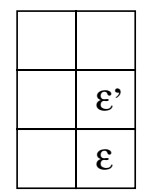

(b)

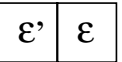

(c)

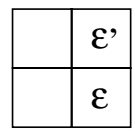

Figure 17. Some small $\epsilon$ cases, with matched box denoted by $\epsilon^{\prime}$

Claim 6.6. (i) There is no box of content $c(\epsilon)$ in $\operatorname{rem}\left(\mu^{\prime}\right)$.

(ii) There is a unique box $\epsilon^{\prime}$ of content $1-\delta-c(\epsilon)$ in $\operatorname{add}\left(\mu^{\prime}\right)$.

(iii) If $\left|\lambda / \mu^{\prime}\right|>2$, then the pair $\lambda-\epsilon$ and $\mu^{\prime}+\epsilon^{\prime}$ is balanced, and the associated skew partition is minimal in the set of those of the form $(\lambda-\epsilon) /\left(\mu+\epsilon^{\prime}\right)^{k}$, with $\epsilon_{k}$ in $\operatorname{rem}\left((\lambda-\epsilon) /\left(\mu^{\prime}+\epsilon^{\prime}\right)\right)$. Equivalently, for every $\epsilon_{k}$ in $\operatorname{rem}\left((\lambda-\epsilon) /\left(\mu^{\prime}+\epsilon^{\prime}\right)\right)$ we have

$$
(\lambda-\epsilon) /\left(\mu+\epsilon^{\prime}\right)^{k}=(\lambda-\epsilon) /\left(\mu+\epsilon^{\prime}\right) .
$$

Before proving this claim, we show how it can be used to complete the proof of Theorem 6.5. Note that if $\lambda-\epsilon$ has a removable box $\tau$ with content $1-\delta-$ $c(\epsilon)$, then by minimality $\lambda / \mu^{\prime}=\{\epsilon, \tau\}$, and we are done by Theorem 4.4 and our assumptions on $\lambda$. Thus we assume that there is no such removable box. By Frobenius reciprocity, Corollary 4.8, and Lemma 4.10, we have

$$
\begin{aligned}
\operatorname{Hom}\left(\Delta_{n}(\lambda), \Delta_{n}\left(\mu^{\prime}\right)\right) & \cong \operatorname{Hom}\left(\operatorname{pr}_{\lambda} \operatorname{ind}_{n-1} \Delta_{n-1}(\lambda-\epsilon), \Delta_{n}\left(\mu^{\prime}\right)\right) \\
& \cong \operatorname{Hom}\left(\Delta_{n-1}(\lambda-\epsilon), \operatorname{pr}_{\lambda-\epsilon} \operatorname{res}_{n} \Delta_{n}\left(\mu^{\prime}\right)\right) .
\end{aligned}
$$

By the first two parts of Claim 6.6 this latter Hom-space is isomorphic to

$$
\operatorname{Hom}\left(\Delta_{n-1}(\lambda-\epsilon), \Delta_{n-1}\left(\mu^{\prime}+\epsilon^{\prime}\right)\right)
$$

and by the final part of Claim 6.6 (and induction) this is non-zero as required.

Thus it only remains to prove Claim 6.6.

Proof of Claim 6.6. (i) First suppose that there is only one box in $\lambda / \mu^{\prime}$ with content $c(\epsilon)$. By construction, if there are any boxes above $\epsilon$ in $\lambda / \mu^{\prime}$, then the one with largest content, or its matched pair, is removable. But this contradicts the choice of $\epsilon$. The other possibility is that there is a second box $\tau$ in $\lambda / \mu^{\prime}$ with content $c(\epsilon)$, occupying the opposite corner of a two by two square. Arguing as in the previous case, if there are any boxes in $\lambda / \mu^{\prime}$ above this square, then this contradicts the choice of $\epsilon$. These two cases are illustrated in Figure 18(a) and (b). In both these cases we deduce that $\mu^{\prime}$ cannot have a removable box of content $c(\epsilon)$, as there must be boxes to the right of any such box in $\mu^{\prime}$.

(a)

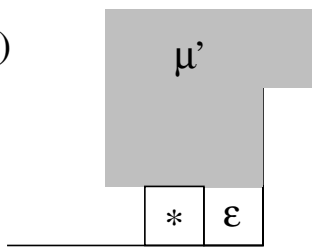

(b)

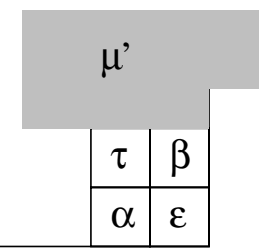

Figure 18. Two corner cases 
(ii) Note that if $\lambda / \mu^{\prime}$ consists of two boxes, then the result is obvious, so we assume this is not the case. It is also clear that any addable box of a given content must be unique. Let $\epsilon^{\prime}$ be the maximal box in $\lambda / \mu^{\prime}$ with content $1-\delta-c(\epsilon)$.

First suppose that $\lambda / \mu^{\prime}$ has only one box with content $c(\epsilon)$, so that we are in the case shown in Figure 18(a). The box $*^{\prime}$ paired with $*$ in Figure 18(a) must be to the right or above $\epsilon^{\prime}$, and hence we are in one of the two configurations shown in Figure 19

(a)

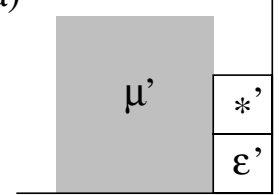

(b)

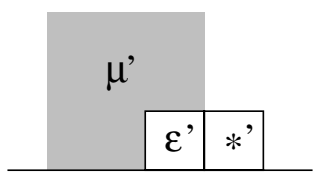

Figure 19. The first corner case

The case in Figure 19(a) is impossible by our assumption on the size of $\lambda / \mu^{\prime}$ (and minimality), as both $\epsilon$ and $\epsilon^{\prime}$ are removable boxes. In the remaining case it is clear that $\mu^{\prime}$ has addable box $\epsilon^{\prime}$, as required.

Next suppose that $\lambda / \mu^{\prime}$ has two boxes with content $c(\epsilon)$, so that we are in the case shown in Figure 18(b). As in the previous case, the box $\alpha^{\prime}$ paired with $\alpha$ must be to the right or above $\epsilon^{\prime}$. If it is above, then we have a configuration similar to that in Figure 19(a), and hence $\epsilon^{\prime}$ is a removable box. But this is impossible exactly as for the case in Figure 19(a). Hence $\alpha^{\prime}$ must be to the right of $\epsilon^{\prime}$, and we must have a configuration as in Figure 20. But this configuration clearly has an addable box, $\tau^{\prime}$, of content $c\left(\epsilon^{\prime}\right)$.

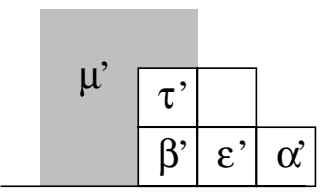

Figure 20. The second corner case

(iii) The two partitions $\lambda-\epsilon$ and $\mu^{\prime}+\epsilon^{\prime}$ are clearly balanced. For minimality we consider the various cases that can arise. If we are in the case shown in Figure 18(a), then paired boxes are as shown in Figure 19(b). Suppose for a contradiction that $(\lambda-\epsilon) /\left(\mu^{\prime}+\epsilon^{\prime}\right)$ is not minimal, and hence contains a smaller skew partition $\eta$. If $\eta$ does not involve $*$ and $*^{\prime}$, then it is also contained in $\lambda / \mu^{\prime}$, which contradicts the minimality of this original pair. If $\eta$ does involve $*$ and $*^{\prime}$, then this contradicts $\lambda / \mu^{\prime}$ being minimal, as $\lambda / \mu^{\prime}$ contains $\eta \cup\left\{*, *^{\prime}\right\}$, which is a smaller sub-skew partition of $\lambda / \mu^{\prime}$.

Now consider the case shown in Figure 18(b), where the paired boxes are as in Figure 20. As before, suppose for a contradiction that $(\lambda-\epsilon) /\left(\mu^{\prime}+\tau^{\prime}\right)$ is not minimal, and hence contains a smaller skew partition $\eta$. If $\eta$ does not involve $\alpha$ and $\alpha^{\prime}$, then it is also contained in $\lambda / \mu^{\prime}$. If $\eta$ does involve $\alpha$ and $\alpha^{\prime}$ but not $\tau$ and $\epsilon^{\prime}$, then $\eta \cup\left\{\epsilon, \epsilon^{\prime}\right\}$ is a removable skew inside $\lambda / \mu^{\prime}$. Finally, if $\eta$ involves all of $\alpha$, $\alpha^{\prime}, \tau$, and $\epsilon^{\prime}$, then $\eta$ must also involve $\beta$ and $\beta^{\prime}$. Now the skew obtained from $\eta$ by 
replacing $\tau$ by $\epsilon$ can be removed from $\lambda / \mu^{\prime}$. In each of these three cases we have found a proper removable skew inside $\lambda / \mu^{\prime}$, which contradicts the minimality of $\lambda / \mu^{\prime}$. Thus $(\lambda-\epsilon) /\left(\mu^{\prime}+\tau^{\prime}\right)$ must be minimal, which completes the proof of Claim 6.6, and hence also of Theorem 6.5.

Corollary 6.7. Two weights $\lambda$ and $\mu$ are in the same block of $B_{n}$ if and only if they are balanced. Each block contains a unique minimal weight.

Proof. In Corollary 4.8 we proved that two weights in the same block must be balanced. For the reverse implication, we will proceed by induction. By Theorem 6.5. if $\lambda$ contains a smaller partition $\mu$ with which it is balanced, then there exists some $\mu^{\prime} \subset \lambda$ with a non-zero homomorphism from $\Delta_{n}(\lambda)$ to $\Delta_{n}\left(\mu^{\prime}\right)$. In particular, $\lambda$ and $\mu^{\prime}$ will lie in the same block of $B_{n}$. Thus it is enough to show that there is a unique minimal partition in the set of partitions which are balanced with $\lambda$.

But if there are two such minimal partitions $\mu$ and $\nu$, then set $\eta=\mu \cap \nu$. Clearly, $\eta$ is a partition, and it forms a balanced pair with both $\mu$ and $\nu$ (and hence with $\lambda)$. This contradicts our assumption of minimality

We conclude this section with a description of the minimal partitions in each block (and hence give a parametrisation of the blocks). We begin by constructing inductively a skew partition $\hat{\lambda}$ related to $\lambda$. Let $\lambda(0)=\lambda$. Given $\lambda(i)$, consider $\epsilon \in \operatorname{rem}(\lambda(i))$ such that $\left|c(\epsilon)-\frac{1-\delta}{2}\right|$ is maximal. Suppose that there does not exist $\epsilon^{\prime} \in[\lambda]$ with $c(\epsilon)+c\left(\epsilon^{\prime}\right)=1-\delta$ and $\epsilon^{\prime} \neq \epsilon$. Hence either the set of rows $\lambda^{t}$ above and including the row containing $\epsilon$ (if $c(\epsilon)-\frac{1-\delta}{2}>0$ ) or the set of columns $\lambda^{l}$ to the the left of and including the column containing $\epsilon$ (if $c(\epsilon)-\frac{1-\delta}{2}<0$ ) cannot be removed. In this case set $\lambda(i+1)=\lambda(i) / \lambda^{t}$, respectively, $\lambda(i+1)=\lambda(i) / \lambda^{l}$. If there exists $\epsilon^{\prime} \in[\lambda]$ with $c(\epsilon)+c\left(\epsilon^{\prime}\right)=1-\delta$ and $\epsilon^{\prime} \neq \epsilon$, then $\hat{\lambda}=\lambda(i)$. This procedure will eventually terminate in the construction of $\hat{\lambda}$.
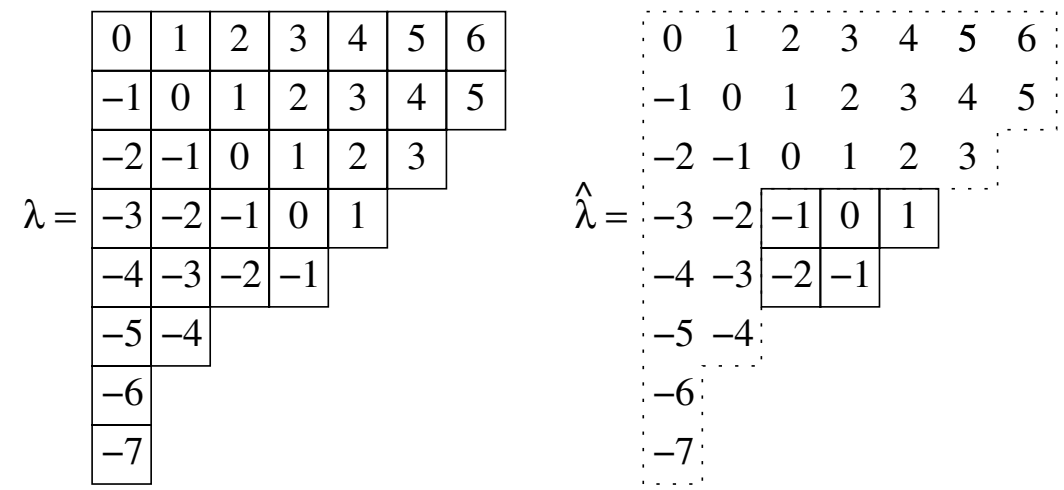

Figure 21. An example of the construction of $\hat{\lambda}$

Example 6.8. As an example of this construction, consider $\delta=1$ and $\lambda=$ $\left(7^{2}, 6,5,4,2,1^{2}\right)$, as illustrated in Figure 21. At the first stage, we take $\epsilon$ to be the box labelled -7 , and hence remove the first column. Next we take the box labelled 5, and remove the first two rows. This is followed by the removal of the 
second column, then the third row, leaving the skew partition illustrated in the figure. As the two remaining removable nodes both have a paired partner (in this case each other) no more rows or columns need be removed, and we have constructed $\hat{\lambda}$.

Proposition 6.9. The minimal partitions in each block are precisely those for which either $\hat{\lambda}=\emptyset$ or a single row or column, or $\delta$ is even and $\hat{\lambda}$ consists of two rows, the second of which has final box of content $-\frac{\delta}{2}$.

Proof. Clearly, if $\hat{\lambda}=\emptyset$, then $\lambda$ is minimal in its block. In the remaining cases, removal of any part of $\lambda$ can only involve boxes in $\hat{\lambda}$, and hence to be balanced must involve either a single unpaired box of content $\frac{1-\delta}{2}$ or a single vertical pair in the configuration shown in Figure 5(b). But this is impossible. Hence we assume that $\hat{\lambda}$ is not of the form given in the proposition, and will show that $\lambda$ is not minimal.

First suppose that $\delta$ is odd. If $\hat{\lambda}$ contains two boxes of content $\frac{1-\delta}{2}$, then we can construct a maximal balanced subpartition of $\lambda$, mimicking the process in Definitions 6.1 and 6.3 by starting with $\epsilon$. Hence by Theorem 6.5 $\lambda$ is non-minimal. If $\hat{\lambda}$ only contains one box $\omega$ with content $\frac{1-\delta}{2}$, then again, by considering Definitions 6.1 and 6.3 and Theorem 6.5, any removable balanced skew-partition must involve $\omega$. The assumption also implies that $\epsilon$ is in the first row or column of $\hat{\lambda}$.

Suppose that $\epsilon$ is in the first row of $\hat{\lambda}$ and there is more than one row (the case where $\epsilon$ is in the first column is similar). If $\lambda$ is minimal, then no final segment of this row has a removable paired segment in $\hat{\lambda}$; this can only arise if $\hat{\lambda}$ is of the form shown in Figure 22 (where shaded areas indicate boxes definitely not in $\hat{\lambda}$ ), where $\tau$ is not paired with any box to the right of $\omega$. But this means that $\tau$ has content $\frac{1-\delta}{2}$ which is impossible, and hence $\lambda$ is not minimal.

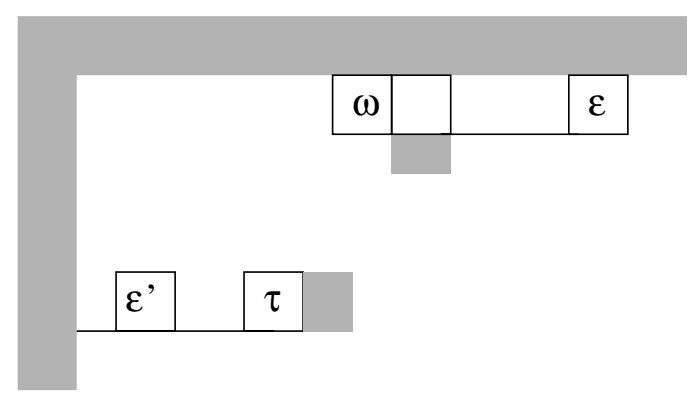

Figure 22. Possible configuration of $\hat{\lambda}$ when $\delta$ is odd

Now suppose that $\delta$ is even. If $\hat{\lambda}$ contains either of the configurations shown in Figure 23(a) and (b) then we can again construct a maximal balanced subpartition, and by Theorem $6.5 \lambda$ is not minimal.

If $\hat{\lambda}$ contains only one box with content either $-\frac{\delta}{2}$ or $1-\frac{\delta}{2}$, then this box is either at the end of the first row or bottom of the first column, which contradicts the definition of $\hat{\lambda}$. Thus we must have one of the configurations in Figure 23(c) or (d).

In case (c) $\epsilon$ must lie at the end of the first column, and in case (d) at the end of the first row. Arguing as in the $\delta$ odd case, we see in case (c) that if $\lambda$ is minimal, then $\hat{\lambda}$ must consist of a single column. However, in case (d), if $\lambda$ is minimal, then 
(a)

\begin{tabular}{|r|r|}
\hline $1-\underline{\delta}$ & $-\underline{\delta}$ \\
\hline
\end{tabular}

(b)

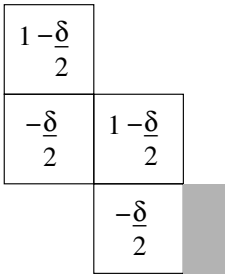

(c)

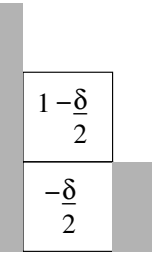

(d)

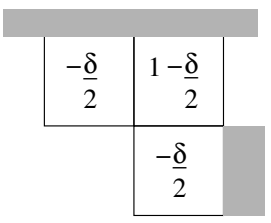

Figure 23. Possible configurations in $\hat{\lambda}$ when $\delta$ is even

we either have a single row or we are in a similar situation to that in Figure 22 and $\tau$ must have content $-\frac{\delta}{2}$. But this implies that $\hat{\lambda}$ consists of two rows with the final box of the second row having content $-\frac{\delta}{2}$, which contradicts our assumptions on $\lambda$.

Thus the only cases where $\lambda$ is a minimal partition are those described in the theorem, and so we are done.

Example 6.10. To illustrate the last result, consider $\delta=1$ with $\lambda=\left(7,6^{2}, 5,2^{2}\right)$ as shown in Figure 24. The associated $\hat{\lambda}$ is also shown, and has only one row, and it is easy to see that $\lambda$ is indeed minimal inside its block.
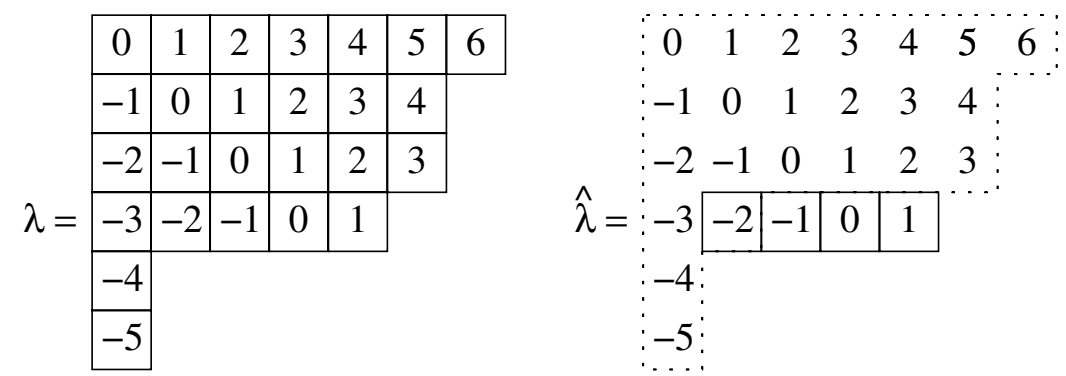

Figure 24. A minimal weight $\lambda$ and the associated $\hat{\lambda}$

\section{ON THE SUBMOdULE STRUCTURE OF CERTAIN STANDARD MODULES}

In this section we will show that the structure of standard modules can become arbitrarily complicated (as measured by their Loewy length and number of simple modules in each Loewy layer). For this it will be sufficient to consider certain special partitions which can be more easily analysed.

Lemma 7.1. If $\epsilon_{i} \in \operatorname{rem}(\lambda)$, then

$$
\left[\operatorname{res}_{n} L_{n}(\lambda): L_{n-1}\left(\lambda-\epsilon_{i}\right)\right] \neq 0 .
$$

Proof. By (2.2) and (2.4) we may assume that $\lambda \vdash n$; the result then follows from Proposition 2.7.

When considering a multi-skew-partition of differences these skew partitions will be listed in the order from top right to bottom left. We will extend the power notation for partitions to multipartitions, so $\left((2)^{2},\left(21^{3}\right)\right)$ will denote the triple of partitions (2), (2), and $\left(21^{3}\right)$. 
Example 7.2. To illustrate these definitions we return to the partitions $\lambda$ and $\mu$ considered in Figure 7 In this case we have $\operatorname{add}(\lambda)=\{-5,-3,-1,3,6\}$ and $\operatorname{rem}(\lambda)=\{-4,-2,1,5\}$. Similarly, $\operatorname{add}(\mu)=\{-3,1,5\}$ and $\operatorname{rem}(\mu)=\{-1,4\}$. The pair $(\lambda, \mu)$ is not $\delta$-balanced for any $\delta$, and $\lambda /(\lambda \cap \mu)$ has shape $\left((1),\left(2^{2}\right),(2,1)\right)$.

We will be interested in $\delta$-balanced pairs $\mu \subset \lambda$ such that the associated skew partition consists entirely of isolated boxes. If $\mu \subset \lambda$ are balanced with $\lambda / \mu=\left((1)^{2 m}\right)$, denote the matched pairs of boxes in $\lambda / \mu$ by $\epsilon_{1}, \epsilon_{1}^{\prime}, \ldots, \epsilon_{m}, \epsilon_{m}^{\prime}$ with respective contents $a_{1}, a_{1}^{\prime}, \ldots, a_{m}, a_{m}^{\prime}$. Let $\mathcal{P}(m)$ denote the power set of $\{1,2, \ldots, m\}$, and for $x \in \mathcal{P}(m)$ set

$$
\lambda-x=\lambda-\sum_{i \in x}\left(\epsilon_{i}+\epsilon_{i}^{\prime}\right) .
$$

For example, $\lambda-\{1, \ldots, m\}=\mu$.

Theorem 7.3. Let $\lambda \vdash n$ and $\mu \subset \lambda$ be a balanced pair with $\lambda / \mu=\left((1)^{2 m}\right)$. Then

$$
\operatorname{dim} \operatorname{Hom}\left(\Delta_{n}(\lambda), \Delta_{n}(\mu)\right)=1
$$

and

$$
\left[\Delta_{n}(\mu): L_{n}(\lambda-x)\right]=1
$$

for all $x \in \mathcal{P}(m)$

Further, denote by $\mathcal{L}(\mu, \lambda)$ the induced lattice in the full submodule lattice of $\Delta_{n}(\mu)$ with vertices those simple modules of the form $L_{n}(\lambda-x)$ for some $x \in \mathcal{P}(m)$. Then $\mathcal{L}(\mu, \lambda)$ is isomorphic to the superset lattice on $\mathcal{P}(m)$; i.e. every submodule of $\Delta_{n}(\mu)$ which contains $L_{n}(\lambda-x)$ contains $L_{n}(\lambda-y)$ for all $y \subset x$.

In particular, the length of the socle series of $\Delta_{n}(\mu)$ is at least $m+1$ and there is a socle series layer containing at least $m$ simple modules.

Remark 7.4. (i) Note that for the induced lattice we are only considering factors of the form $L_{n}(\lambda-x)$. In general the module $\Delta_{n}(\mu)$ may have many other composition factors. Thus an arrow $A \rightarrow B$ in our induced lattice structure is to be understood as representing some non-trivial extension in $\Delta_{n}(\mu)$ with $A$ in the head and $B$ in the socle.

(ii) Clearly the final part of the theorem can be strengthened, but it is already enough to show that standard modules can have arbitrarily large socle series lengths (and layers of arbitrary width).

Example 7.5. If $\lambda$ and $\mu$ are balanced with $\lambda / \mu=\left((1)^{6}\right)=\left\{\epsilon_{1}, \epsilon_{1}^{\prime}, \epsilon_{2}, \epsilon_{2}^{\prime}, \epsilon_{3}, \epsilon_{3}^{\prime}\right\}$, then the lattice $\mathcal{L}(\mu, \lambda)$ is illustrated in Figure 25.

Proof. We proceed by induction on $m$, the result being obvious for $m=0$. By Frobenius reciprocity we have

$$
\operatorname{Hom}\left(\operatorname{ind}_{n-1} \Delta_{n-1}\left(\lambda-\epsilon_{i}\right), \Delta_{n}(\mu)\right) \cong \operatorname{Hom}\left(\Delta_{n-1}\left(\lambda-\epsilon_{i}\right), \operatorname{res}_{n} \Delta_{n}(\mu)\right) .
$$

By Proposition 2.7 and Corollary 4.8 the only submodule of $\operatorname{res}_{n} \Delta_{n}(\mu)$ which can lie in the same block as $\Delta_{n-1}\left(\lambda-\epsilon_{i}\right)$ is isomorphic to $\Delta_{n-1}\left(\mu+\epsilon_{i}^{\prime}\right)$, and hence by the inductive hypothesis the right-hand side of (7.1) is one-dimensional. Lemma 4.10 now implies that $L_{n}(\lambda)$ is a composition factor of $\Delta_{n}(\mu)$. To show that $\operatorname{dim} \operatorname{Hom}\left(\Delta_{n}(\lambda), \Delta_{n}(\mu)\right)=1$ it will be enough to show that there is precisely one copy of this composition factor in $\Delta_{n}(\mu)$ (which will necessarily lie in the socle). 


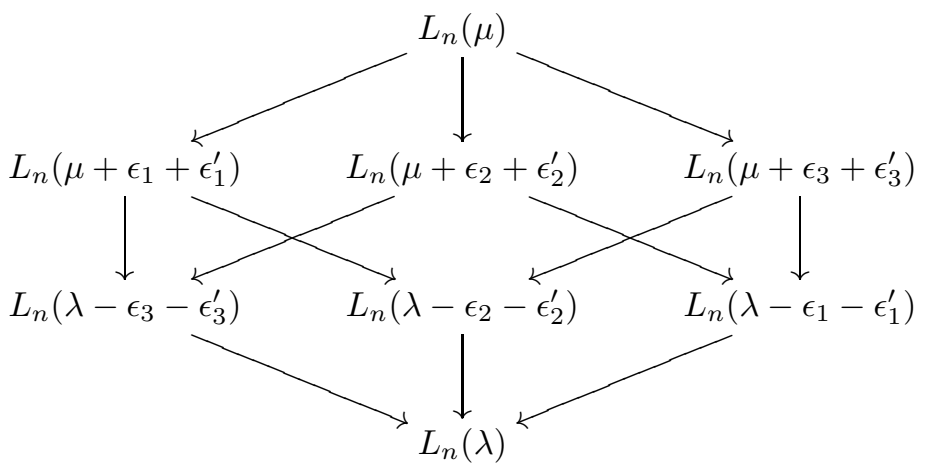

FiguRE 25. An example of $\mathcal{L}(\mu, \lambda)$

By assumption the pair $(\lambda, \mu)$ is balanced. We will define the bias of a pair $(\lambda, \tau)$ with $|\lambda \Delta \tau|=2 t$ to be

$$
b(\lambda, \tau)=\left(\sum_{d \in \lambda \Delta \tau} c(d)\right)-t(1-\delta) .
$$

Thus a balanced pair has zero bias. Consider the restriction $\operatorname{res}_{n} \Delta_{n}(\mu)$. By Proposition 2.7 we have a short exact sequence

$$
0 \rightarrow \bigoplus_{\tau \triangleleft \mu} \Delta_{n-1}(\tau) \rightarrow \operatorname{res}_{n} \Delta_{n}(\mu) \rightarrow \bigoplus_{\tau \triangleright \mu} \Delta_{n-1}(\tau) \rightarrow 0 .
$$

Note that $\mu$ has no removable boxes with content $\pm a_{i}$ for $1 \leq i \leq m$, as this would contradict the existence of an addable node with such a content. Thus the only modules $\Delta_{n-1}(\tau)$ in the sequence (7.2) with bias $\pm a_{i}$ are $\Delta_{n-1}\left(\mu+\epsilon_{i}\right)$ and $\Delta_{n-1}\left(\mu+\epsilon_{i}^{\prime}\right)$

By Lemma 7.1 we have that

$$
\left[\operatorname{res}_{n} L_{n}(\lambda-x): L_{n-1}\left(\lambda-x-\epsilon_{i}\right)\right]=1
$$

provided that $i \notin x$. But (by the observations on the bias above) $L_{n-1}\left(\lambda-x-\epsilon_{i}\right)$ can only occur in $\Delta_{n-1}\left(\mu+\epsilon_{i}^{\prime}\right)$, and by the inductive hypothesis it occurs there precisely once. By varying $i$ we deduce that there is at most one copy of each $L_{n}(\lambda-x)$ in $\Delta_{n}(\mu)$. But by induction we know that there is a homomorphism from $\Delta_{n^{\prime}}(\lambda-x)$ to $\Delta_{n^{\prime}}(\mu)$ where $n^{\prime}=|\lambda-x|$, and hence by repeated applications of $G$ that there is a homomorphism from $\Delta_{n}(\lambda-x)$ to $\Delta_{n}(\mu)$. Hence we see that $L_{n}(\lambda-x)$ occurs exactly once in $\Delta_{n}(\mu)$.

Now consider the summand $\Delta_{n-1}\left(\mu+\epsilon_{i}^{\prime}\right)$ in $\operatorname{res}_{n} \Delta_{n}(\mu)$. This is the only summand of the restriction in which $L_{n-1}\left(\lambda-x-\epsilon_{i}\right)$ (with $i \notin x$ ) can arise, and this simple module appears in an extension below $L_{n-1}\left(\lambda-y-\epsilon_{i}\right)$ for all $y \supset x$ (with $i \notin y)$, by the inductive hypothesis. In particular the copy of $L_{n-1}\left(\lambda-x-\epsilon_{i}\right)$ appearing in $\operatorname{res}_{n} L_{n}(\lambda-x)$ appears below $L_{n-1}\left(\lambda-x-\epsilon_{i}-\epsilon_{j}-\epsilon_{j}^{\prime}\right)$ in an extension, and this latter simple module must come from $\operatorname{res}_{n} L_{n}\left(\lambda-x-\epsilon_{j}-\epsilon_{j}^{\prime}\right)$. It follows that $L_{n}(\lambda-x)$ must occur in some extension beneath $L_{n}\left(\lambda-x-\epsilon_{j}-\epsilon_{j}^{\prime}\right)$. This argument works for all $j$ and $x$, and hence verifies the claimed submodule 
structure except for the top two layers. However, these are forced by the structure of standard modules.

\section{THE CASE $\delta=0$}

In this section we will sketch the modifications to the preceding arguments which are required when $\delta=0$. The most obvious change is that the idempotents $e_{n}$ considered thus far no longer exist. Related to this is the failure of the algebras to be quasi-hereditary when $n$ is even.

The first two axioms in the tower of recollement formalism in CMPX06 use quasi-heredity to define a family of standard modules for each algebra in the tower, together with associated idempotents that provide localisation and globalisation functors between the various module categories. The remaining axioms require that this structure be compatible with induction and restriction, and that the standard modules have a local behaviour under induction and restriction.

The key facts that we use from the general theory of quasi-hereditary algebras are: (i) that all simple modules occur as the head of a unique standard module; (ii) that all other composition factors are lower in some order coming from the heredity chain; (iii) that the standard modules are mapped to standards (or zero) via localisation and globalisation; (iv) that the blocks of the algebra are given by the equivalence relation generated by the relation of being common composition factors of a standard module.

We can replace the quasi-hereditary formalism by any other which provides of properties (i-iv) above, and provided this satisfies all of the remaining compatibility and locality axioms for induction and restriction we will be able to proceed as for a tower of recollement.

One candidate for such a replacement is the theory of cellular algebras developed by Graham and Lehrer GL96, where the role of standard modules is played by cell modules. In this setting (i) does not hold; although all simple modules do arise in this manner we no longer necessarily obtain a distinct simple module from each cell module. (ii) and (iv) do hold for cell modules, but in general we do not expect to have idempotents associated to the defining cell chain, and so we cannot use localisation and globalisation as above in (iii).

Fortunately, for the Brauer algebras these problems can all be resolved. However, this is at the expense of some addition calculations (which is one reason why we prefer to use the quasi-hereditary framework for the bulk of this paper). Also the quasi-hereditary cases have better homological properties, and should have interesting Ringel duals (which are not available in the cellular setting).

We will finish by indicating the modifications that are needed in the cellular case. The cellularity of Brauer algebras was first shown in GL96]; however, for our purposes the alternative approach to cellularity in [KX99], together with the explicit demonstration of this for the Brauer algebras in [KX01, will be more convenient.

For $n \geq 3$ let $\bar{e}_{n}$ be the element illustrated in Figure 26. This is an idempotent for every value of $\delta$, and satisfies (A1), i.e. $\bar{e}_{n} B_{n} \bar{e}_{n} \cong B_{n-2}$.

This allows us to define localisation and globalisation functors $\bar{F}_{n}$ and $\bar{G}_{n}$ except for $\bar{F}_{2}$ and $\bar{G}_{0}$. The cell modules are explicitly described in [KX01] and it is easy to verify that this description coincides with that given for standard modules in Lemma 2.4 (and with the modules considered in DWH99). Furthermore, an easy calculation shows that $\bar{F}_{n}$ and $\bar{G}_{n}$ take cell modules to cell modules or zero, which 


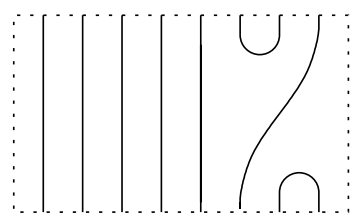

Figure 26. The element $\bar{e}_{n}$ in $B_{n}$

gives the desired analogue of condition (iii) above. As our bases do not depend on $\delta$, the remaining results in Section 2 on standard modules go through unchanged.

The simple modules no longer have the same parametrisation as the cell modules (unlike the quasi-hereditary case). However, we can inductively parametrise them as in Section 2 using the idempotents $\bar{e}_{n}$, except that instead of reducing the calculation entirely to the various symmetric groups, we also have to consider the algebra $B_{2}(0)$ directly. This is due to the lack of localisation and globalisation functors relating $B_{0}(0)$ and $B_{2}(0)$.

However, the latter is only a three-dimensional algebra, and so the structure of its cell modules is easy to determine by hand. The cell modules $\Delta_{2}(0)$ and $\Delta_{2}(2)$ are easily seen to be isomorphic (and simple). This case gives the one difference in our parametrisation of simple modules compared to the quasi-hereditary case, as we no longer obtain a simple module corresponding to the empty partition. (Our choice of labelling here of the simple $L_{2}(2)$ is so that the effect of losing one simple module is just a degeneration of the usual case, and does not effect our partial order.)

All of the remaining results in Sections 3-7 rely on properties (i-iv) above or explicit calculations using the bases of standard modules. As we now have cellular versions of (i-iv) and the same bases for cell modules, these all generalise to give corresponding results for $\delta=0$.

\section{REFERENCES}

[Bra37] R. Brauer, On algebras which are connected with the semisimple continuous groups, Ann. of Math. 38 (1937), 857-872. MR1503378

[Bro55] W. Brown, An algebra related to the orthogonal group, Michigan Math. J. 3 (1955), 1-22. MR0072122 (17:232a)

[CDM] A. G. Cox, M. De Visscher, and P. P. Martin, A geometric characterisation of the blocks of the Brauer algebra, J. London Math. Soc, to appear.

[CMPX06] A. G. Cox, P. P. Martin, A. E. Parker, and C. Xi, Representation theory of towers of recollement: theory, notes, and examples, J. Algebra 302 (2006), 340-360. MR2236606 (2007b:16028)

[DDH08] R. Dipper, S. Doty, and J. Hu, Brauer's centralizer algebras, symplectic Schur algebras and Schur-Weyl duality, Trans. Amer. Math. Soc. 360 (2008), 189-213. MR.2342000 (2008g:16032)

[DH09] S. Doty, and J. Hu, Schur-Weyl duality for orthogonal groups, Proc. London Math. Soc., 98 (2009), 679-713.

[Dia88] P. Diaconis, Group representations in probability and statistics, Institute of Mathematical Statistics Lecture Notes, Monograph Series 11, Institute of Mathematical Statistics, 1988. MR964069 (90a:60001)

[Don98] S. Donkin, The q-Schur algebra, London Math. Soc. Lecture Notes Series, vol. 253, Cambridge University Press, 1998. MR 1707336 (2001h:20072)

[Dot98] S. Doty, Polynomial representations, algebraic monoids, and Schur algebras of classical type, J. Pure Appl. Algebra 123 (1998), 165-199. MR.1492900 (98j:20057) 
[DT] S. Donkin and R. Tange, The Brauer algebra and the symplectic Schur algebra, Math. Z., to appear.

[DWH99] W. F. Doran, D. B. Wales, and P. J. Hanlon, On the semisimplicity of the Brauer centralizer algebras, J. Algebra 211 (1999), 647-685. MR.1666664 (99k:16034)

[Ful97] W. Fulton, Young tableaux, London Math. Soc. Student Texts, vol. 35, Cambridge, 1997. MR1464693 (99f:05119)

[GL96] J. J. Graham and G. I. Lehrer, Cellular algebras, Invent. Math. 123 (1996), 1-34. MR.1376244 (97h:20016)

[Gre80] J. A. Green, Polynomial representations of $G L_{n}$, Lecture Notes in Mathematics 830, Springer, 1980. MR606556 (83j:20003)

[HP06] R. Hartmann and R. Paget, Young modules and filtration multiplicities for Brauer algebras, Math. Z. 254 (2006), 333-357. MR2262706 (2008j:20009)

[HW89a] P. J. Hanlon and D. B. Wales, Eigenvalues connected with Brauer's centralizer algebras, J. Algebra 121 (1989), 446-476. MR.992776 (91a:20041b)

[HW89b] - On the decomposition of Brauer's centralizer algebras, J. Algebra 121 (1989), 409-445. MR992775 (91a:20041a)

[HW90] - Computing the discriminants of Brauer's centralizer algebras, Math. Comp. 54 (1990), 771-796. MR1010599 (90k:20076)

[HW94] A tower construction for the radical in Brauer's centralizer algebras, J. Algebra 164 (1994), 773-830. MR1272114 (95f:20070)

[Jam78] G. D. James, The representation theory of the symmetric groups, Lecture Notes in Mathematics, vol. 682, Springer, 1978. MR513828 (80g:20019)

[JK81] G. D. James and A. Kerber, The representation theory of the symmetric group, Encyclopedia of Mathematics and its Applications, vol. 16, Addison-Wesley, 1981.

[KX99] S. König and C. Xi, Cellular algebras: Inflations and Morita equivalences, J. London Math. Soc. 60 (1999), 700-722. MR1753809 (2001a:16020)

[KX01] S. König and C. Xi, A characteristic free approach to Brauer algebras, Trans. Amer. Math. Soc. 353 (2001), 1489-1505. MR1806731(2002f:16039)

[Mar91] P. P. Martin, Potts models and related problems in statistical mechanics, World Scientific, Singapore, 1991. MR:1103994 (92m:82030)

[Mar96] , The structure of the Partition algebras, J. Algebra 183 (1996), 319-358. MR1399030 (98g:05152)

[Mat99] A. Mathas, Iwahori-Hecke algebras and Schur algebras of the symmetric groups, University lecture series, vol. 15, American Mathematical Society, 1999. MR.1711316 (2001g:20006)

[MRH04] P. P. Martin and S. Ryom-Hansen, Virtual algebraic Lie theory: Tilting modules and Ringel duals for blob algebras, Proc. London Math. Soc. 89 (2004), 655-675. MR2107010 (2005i:20007)

[MW03] P. P. Martin and D. Woodcock, Generalized blob algebras and alcove geometry, London Math. Soc. J. of Comp. and Math. 6 (2003), 249-296. MR2051586 (2005f:20013)

[Naz96] M. Nazarov, Young's orthogonal form for Brauer's centralizer algebra, J. Algebra 182 (1996), 664-693. MR 1398116 (97m:20057)

[Oeh01] S. Oehms, Centralizer coalgebras, FRT-construction and symplectic monoids, J. Algebra 244 (2001), 19-44. MR1856529 (2002h:16062)

[OR07] R. Orellana and A. Ram, Affine braids, Markov traces and the category $\mathcal{O}$, in Algebraic groups and homogeneous spaces, Tata Inst. Fund. Res. Stud. Math. (2007), 423-473. MR2348913 (2008m:17034)

[Rui05] H. Rui, A criterion on the semisimple Brauer algebras, J. Comb. Theory Ser. A 111 (2005), 78-88. MR2144855 (2006g:16042)

[Wen88] H. Wenzl, On the structure of Brauer's centralizer algebra, Ann. Math. 128 (1988), 173-193. MR951511 (89h:20059)

[Wey46] H. Weyl, The classical groups, their invariants and representations, Princeton University Press, 1946. MR:1488158 (98k:01049) 
Centre for Mathematical Science, City University, Northampton Square, London, EC1V 0HB, ENGLAND.

E-mail address: A.G.Cox@city.ac.uk

Centre for Mathematical Science, City University, Northampton Square, London, EC1V 0HB, ENGLAND

E-mail address: M.Devisscher@city.ac.uk

Centre for Mathematical Science, City University, Northampton Square, London, EC1V 0HB, ENGLAND

Current address: Department of Pure Mathematics, University at Leeds, Leeds, LS2 9JT, England

E-mail address: ppmartin@maths.leeds.ac.uk 